\title{
Collectin-11 detects stress-induced L-fucose pattern to trigger renal epithelial injury
}

\author{
Conrad A. Farrar, ${ }^{1}$ David Tran, ${ }^{1}$ Ke Li, ${ }^{2}$ Weiju Wu, ${ }^{1}$ Qi Peng, ${ }^{1}$ Wilhelm Schwaeble, ${ }^{3}$ Wuding Zhou, ${ }^{1}$ and Steven H. Sacks ${ }^{1}$ \\ ${ }^{1}$ Medical Research Council (MRC) Centre for Transplantation, King's College London, London, United Kingdom. ${ }^{2}$ Core Research Laboratory, The Second Affiliated Hospital, School of Medicine, Xi'an Jiaotong \\ University, Xi'an, Shaanxi, China. ${ }^{3}$ Department of Infection, Immunity and Inflammation, University of Leicester, Leicester, United Kingdom.
}

\begin{abstract}
Physiochemical stress induces tissue injury as a result of the detection of abnormal molecular patterns by sensory molecules of the innate immune system. Here, we have described how the recently discovered C-type lectin collectin-11 (CL-11, also known as CL-K1 and encoded by COLEC11) recognizes an abnormal pattern of L-fucose on postischemic renal tubule cells and activates a destructive inflammatory response. We found that intrarenal expression of CL-11 rapidly increases in the postischemic period and colocalizes with complement deposited along the basolateral surface of the proximal renal tubule in association with L-fucose, the potential binding ligand for CL-11. Mice with either generalized or kidney-specific deficiency of CL-11 were strongly protected against loss of renal function and tubule injury due to reduced complement deposition. Ex vivo renal tubule cells showed a marked capacity for CL-11 binding that was induced by cell stress under hypoxic or hypothermic conditions and prevented by specific removal of L-fucose. Further analysis revealed that cell-bound CL-11 required the lectin complement pathway-associated protease MASP-2 to trigger complement deposition. Given these results, we conclude that lectin complement pathway activation triggered by ligand-CL-11 interaction in postischemic tissue is a potent source of acute kidney injury and is amenable to sugar-specific blockade.
\end{abstract}

\section{Introduction}

Innate immune factors produced by epithelial cells serve defensive and homeostatic functions and also regulate the inflammatory response following physical and biochemical tissue stress $(1,2)$. Collectin-11 (CL-11, also known as CL-K1 and encoded by COLEC11) is a recently described component of innate defense that is synthesized by several types of nonimmune cells, including epithelial cells of the renal tract and mucosal cells in the intestinal and bronchial tracts $(3,4)$. The collectin family of innate immune molecules to which CL-11 has been assigned (5) are soluble C-type lectins involved in pattern recognition and include mannan-binding lectin (MBL) and the surfactant proteins SP-A and SP-D $(6,7)$.

The structure of CL-11 consists of a globular head, followed by a neck region and an $\mathrm{N}$-terminal collagenous domain (5). Each chain associates to form trimeric CL-11 subunits that combine to give CL-11 oligomers (100-200 kDa). This oligomerization contributes to the high avidity of interaction with monosaccharide ligands. The globular head of CL-11 contains a carbohydrate recognition domain (CRD), which is reported to preferentially bind L-fucose among other possible ligands (5). The collagenous region of CL-11 contains a binding motif for the MBL-associated serine proteases MASP-1, MASP-2, and MASP-3 $(3,8)$, whose functions can initiate the complement cascade but may also involve substrates other than complement $(9,10)$. Like MBL, CL-11 can interact with the surfaces of bacteria, viruses, and fungi, which

Authorship note: C.A. Farrar and D. Tran are co-first authors. W. Zhou and S.H. Sacks are equal senior authors.

Conflict of interest: The authors have declared that no conflict of interest exists. Submitted: May 29, 2015; Accepted: February 24, 2016.

Reference information: J Clin Invest. 2016;126(5):1911-1925. doi:10.1172/JCI83000. indicates a significant role for CL-11 in host defense $(3,8,11)$. However, there are certain biological differences between CL-11 and MBL that could affect the respective roles of these molecules at different tissue sites. The plasma concentration of CL-11 $(12,13)$ is approximately one-tenth the concentration reported for MBL $(2 \mu \mathrm{g} / \mathrm{ml})$, albeit in a different study population (14). In addition, whereas MBL mainly originates by hepatocyte synthesis (15), CL-11 is synthesized at multiple tissue sites including the liver, brain, and heart, as well as in those systems cited above $(3,4,11)$.

CL-11 plays a role in host defense, directing the innate immune response against locally invasive pathogens $(3,8,11)$. A role in embryogenesis is apparent in individuals with inherited deficiency of CL-11, who have craniofacial birth defects and developmental abnormalities of the heart, kidneys, and other tissues (16). As a group, collectins also contribute to the modulation of inflammatory and adaptive immune responses (9, 10). We speculated that, since animal lectins are involved in a broad range of pathophysiological processes, CL-11 could act as an inflammatory trigger through recognition of undefined carbohydrate structures on stressed cells close to the site of CL-11 production. In the present study, we examined the relationship between cell stress, CL-11 production, ligand binding and activation of the innate immune system, using a well-characterized model of renal tubule cell injury caused by induction of hypoxic and hypothermic stress (17-19).

\section{Results}

CL-11 mediates tissue injury following renal stress. To investigate the pathophysiological role of CL-11, we used an established model of ischemic injury with known sensitivity of renal epithelial cells to hypoxic stress and a defined role of complement, in particular 
the central component C3 (20). We induced reperfusion injury in C57BL/6 WT mouse kidneys following a 50-minute period of renal vessel ligation and charted the expression of CL-11 in renal tissue at early time points. This confirmed the basal expression of Colec11 mRNA in normal, nonmanipulated mouse kidney (4) and showed rapid upregulation of CL-11 in the cortex of postischemic kidney, where peak expression of Colec 11 mRNA occurred within 6 hours of reperfusion (Figure 1, A and B). Immunofluorescence localization of CL-11 at 6 hours after reperfusion detected CL-11 within the cytoplasm and on the cell surface of renal tubular epithelial cells (RTECs) (Figure 1C). Tissue staining for C3d, the covalently bound fragment formed after cleavage of C3, showed localization of the activated complement fragment on the RTEC surface (Figure 1C). The merged immunofluorescence image for CL-11 and C3d shows the relationship of these 2 molecules detected at the basal surface of the renal tubules in the hypoxiasensitive zone at the corticomedullary region of the kidney (Figure 1D). In addition, we studied the distribution of L-fucose, a putative ligand for CL-11, whose expression in normal kidney is limited to the cortical tubule segments (21). Our study confirmed this distribution in normal tissue (Supplemental Figure 1; supplemental material available online with this article; doi:10.1172/ JCI83000DS1) and, in ischemic tissue, revealed a disorganized pattern of L-fucose expression, in which L-fucose appeared to be aligned with CL-11 along the basolateral border of the corticomedullary tubules (Figure 1E).

In a second experiment, we compared the ischemic injuries induced in Colec11 $1^{-/}$and WT $\left(\right.$Colec11 $\left.1^{++}\right)$mice to determine the influence of CL-11. On this occasion, we used 50 minutes of ischemia and a longer period of reperfusion ( 24 hours), allowing us to measure the impact of CL-11 at the peak injury time $(18,20)$. We found that $\mathrm{Colec}_{11^{-/}}$mice showed reduced tubule necrosis and inflammatory cell infiltration compared with that seen in Colec11//+ mice (Figure 2, A-H). Specifically, infiltration by neutrophils and macrophages was reduced in the absence of CL-11. In addition, we noted that deposition of complement (C3d) on the kidney tubules of Colec $11^{-/-}$mice was much lower than that observed in Colec $11^{+/+}$mice (Figure 2, I and J), to an extent that was comparable to that of the background staining for $\mathrm{C} 3 \mathrm{~d}$ detected in nonmanipulated Colec11 ${ }^{+/+}$ mouse kidney (Figure 1C and Supplemental Figure 2). Blood urea nitrogen (BUN), a measure of renal function, showed substantial preservation of function in the protected mice (Figure $2 \mathrm{~K}$ ). Thus, CL-11 mediates postischemic tubule damage and interstitial infiltration. However, it was not clear from these data alone whether complement activation played a primary or secondary role.

To determine whether CL-11 participated in the induction of tubule injury or as a reaction to tubule necrosis, we induced a milder period of renal ischemia (30 minutes) in a separate group of mice and, again, evaluated the injury 24 hours after reperfusion. The results showed a good correlation with the severe model (50 minutes of ischemia), since an absence of CL-11 in Colec11 ${ }^{-1}$ mice permitted a less severe loss of renal function, with good preservation of renal architecture and reduced leukocyte infiltration and tubular deposition of complement compared with that seen in Colec11 ${ }^{+/+}$mice (Figure 3, A-I). Furthermore, with the less-intense staining for $\mathrm{C} 3 \mathrm{~d}$ in the $\mathrm{Colec}_{11^{+/+}}$mice using this milder injury model, it was possible to more clearly discern areas along the basal surface of viable renal tubules where CL-11 and C3d were colocated (Figure 3, K and L). We conclude that the early component of postischemic injury was CL-11 dependent and involved concomitant accumulation of C3d on the tubule surface, regardless of tubule necrosis.

In subsequent studies, we combined minimal ischemia (30 minutes) with minimal reperfusion (30 minutes) in order to characterize the distribution of CL-11 and the associated ligand at potential sites targeted by complement, close to the time of the onset of tubule injury. We observed a wide distribution of CL-11 across the cortex and medulla, with maximal ischemia-induced expression in tubules of the inner cortex (Supplemental Figure 3). L-fucose, the putative ligand for CL-11, was limited in expression to the renal cortex (Supplemental Figure 4). Complement activation marked by C3d predominantly occurred on tubules within the renal cortex (Supplemental Figure 5). Finer mapping within the hypoxia-sensitive region of the renal cortex was possible using confocal microscopy with segment-specific markers that differentiated between the proximal and distal segments of renal tubules (Supplemental Figure 6). While CL-11 was expressed in both the proximal tubule (L-fucose-positive, NCC-negative) and distal tubule (L-fucose-negative, NCC-positive) segments in the ischemic cortex, we mainly detected complement activation (C3d) on proximal tubules, where L-fucose and CL-11 were coexpressed. Although distal tubules expressed CL-11, there was no L-fucose expression and no major involvement of complement. Specifically, $94 \%$ of proximal tubules seen in the cross section were C3d positive, while most distal tubule sections (80\%) were C3d negative (Supplemental Figure 6C). In short, complement activation in the ischemia-sensitive zone was selective for tubule segments that expressed both CL-11 and its putative binding ligand.

Our data establish that ischemic renal injury is CL-11 dependent, but do not distinguish between the possible contribution of kidney-derived CL-11 and systemic CL-11 produced by nonrenal sources. To isolate the effect of kidney-derived CL-11, we conducted a renal transplantation study in mice. For kidneys transplanted from Colec11 ${ }^{+/+}$or Colec11 $1^{-/}$donors into syngeneic $\mathrm{Colec}_{11^{+++}}$recipients, we induced cold ischemia (25 minutes), followed by an obligatory period of warm ischemia (30 minutes) during the implantation procedure. In the first group of mice, we removed the kidney 24 hours after transplantation to capture the peak injury for histological analysis. Colec11/-- transplants showed strong protection from renal tubular damage compared with that observed in Colec11 ${ }^{+/+}$control transplants (Figure 4, A and B). In a second group of transplant recipients, we removed the remaining native kidney 6 days after transplantation, so that the subsequent BUN measurement would reflect the function of the transplanted kidney. Although by this stage the renal transplant was recovering from the ischemia-reperfusion insult, we were able to confirm that the function and structure of Colec11 $1^{-/}$transplants were superior to what was observed in the Colec11 $1^{+/+}$controls (Figure 4, C and D). Hence, kidney-derived CL-11 was a determinant of both the peak injury and the rate of recovery following ischemic insult.

Epithelial cell stress causes binding of CL-11. Given the potential link between postischemic injury and kidney-derived CL-11 emerging from the transplantation and localization studies, we explored the specific role of the renal tubule cell. We used pri- 
A

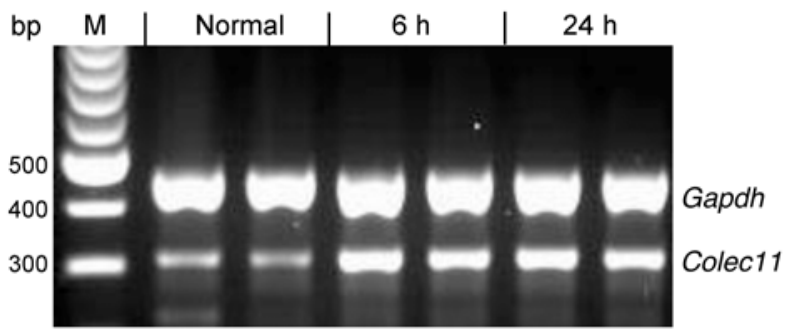

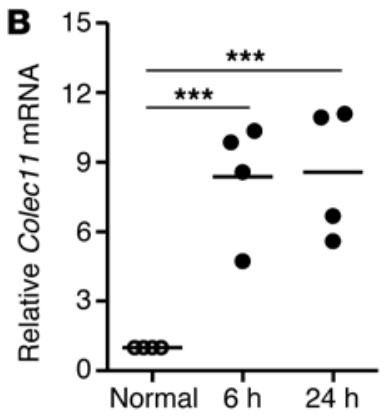

C
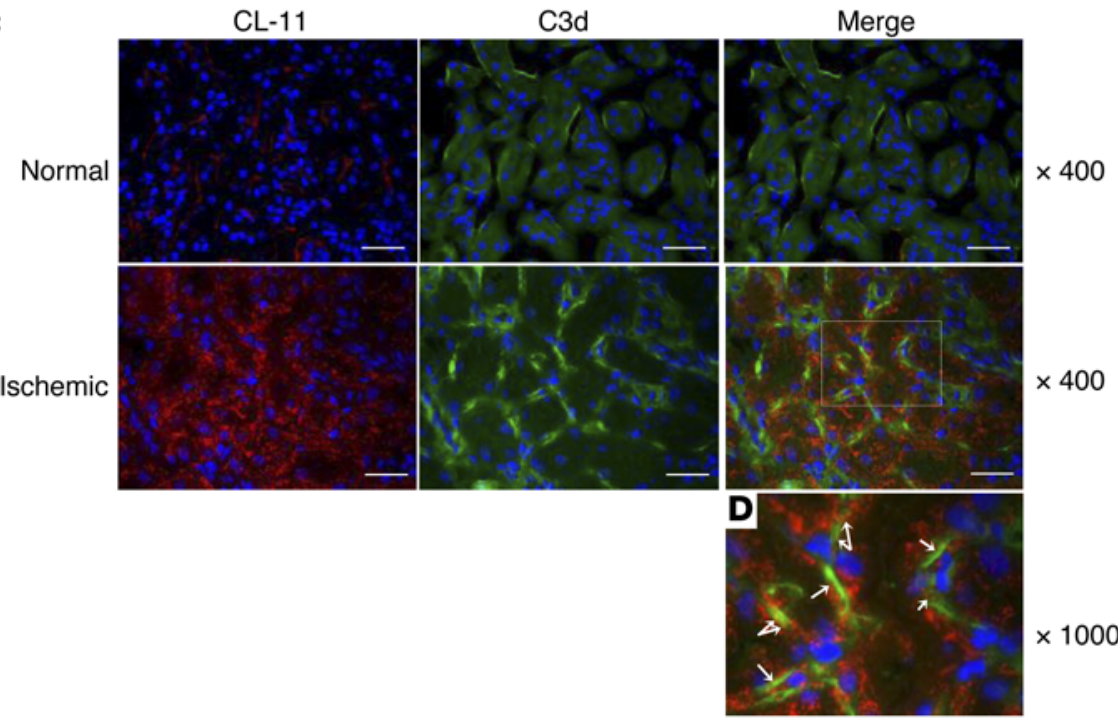

E
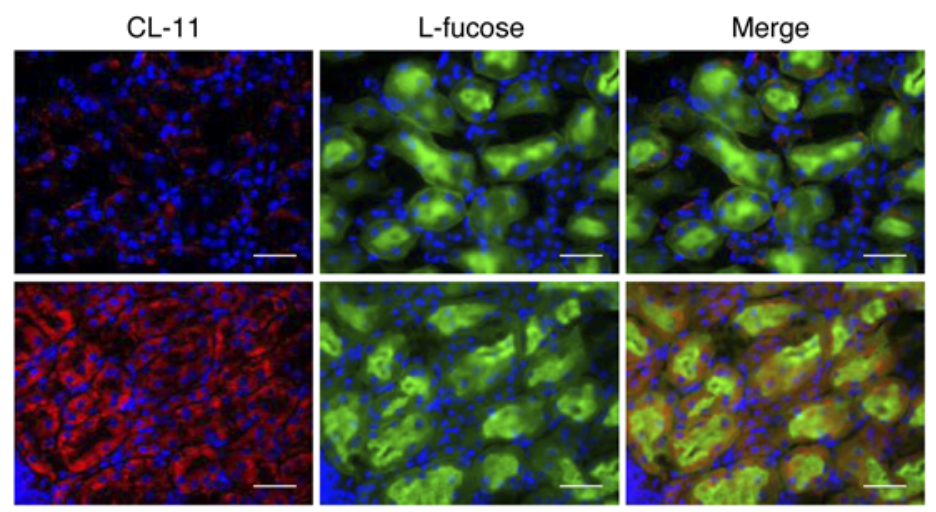

Ischemic

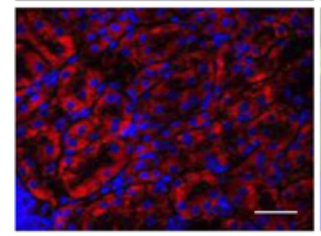

Figure 1. Acute-phase response of CL-11 and associated molecules in mouse kidney. Detection of CL-11 and potential ligand (L-fucose) and complement activation product (C3d) are shown in normal mouse kidney and injured kidney after 50 minutes of ischemia and up to 24 hours of reperfusion. (A) Agarose gel image demonstrating Colec 11 mRNA expression in normal and ischemic kidney tissue, determined by semiquantitative RT-PCR. Colec11 appears as a 315-bp product, and Gapdh (453 bp) is included as an internal control. M, 100 bp DNA ladder. $n=2$ mice/group. (B) Quantification of Colec11 mRNA expression in normal and ischemic kidney tissue by real-time quantitative RT-PCR (qRT-PCR) demonstrating upregulation of intrarenal Colec 11 mRNA expression following ischemic insult. Each dot represents an individual mouse ( $n=4$ mice/ group). ${ }^{* *} P<0.005$, by 1 -way ANOVA. (C) Representative immunofluorescence microscopic images of renal cortex showing particulate staining of $\mathrm{CL}-11$ (red), linear peritubular staining of C3d (green), and a merged image of CL-11 and C3d in normal and ischemic kidney tissue ( $n=4$ mice/group). (D) Enlargement (original magnification, $\times 400$ ) of white boxed area in panel $\mathbf{C}$, with arrows showing coexpression of $\mathrm{CL}-11$ and complement deposition (orange) along the basolateral border of renal tubular epithelium. (E) Representative fluorescence images of CL-11 (red), L-fucose (green), and a merged image of CL-11 and L-fucose in normal and ischemic kidney tissue ( $n=6$ mice/group) showing ischemia-related coexpression of CL-11 and L-fucose (orange) along the basolateral border of the tubules. Scale bars: $25 \mu \mathrm{m}$.

mary cultures of RTECs derived from Colec11 ${ }^{+/+}$mouse kidney and examined CL-11 production and release following hypoxic or hypothermic cell stress. We determined that induction of cell stress in vitro, followed by restoration of normal culture conditions, increased both the intracellular and extracellular content of CL-11 (Figure 5, A-D). Under these conditions, we detected CL-11 on the RTEC at its basal surface using confocal microscopy combined with a cell-surface marker of F-actin to localize the staining on the outer aspect of the cell (Figure 5D). To detect CL-11 released from the cell, we transferred RTEC culture supernatant to stressed, CL-11-deficient RTECs derived from the KO mouse, thereby eliminating the endogenous CL-11 background from the assay. These results showed a clumped and polarized pattern of CL-11 staining indicative of polyvalent binding to the stressed renal cells (Figure 5, E and F). Thus, the RTEC was both a source and a target of CL-11, which, following cell stress, could be detected bound to the basal cell surface.

We considered whether cell stress was sufficient to induce CL-11 binding at a fixed concentration of CL-11. In this case, we used recombinant murine CL-11 (rCL-11) at a physiological concentration and used Colec11 $1^{-1}$ RTECs to detect CL-11 binding, avoiding the background of endogenous CL-11. The clumped and polarized pattern of rCL-11 binding on stressed cells (Figure 6, A and $\mathrm{B}$ ) was reminiscent of that detected with a high concentration of native CL-11 in the culture supernatant of WT cells. We found that binding of CL-11 to the stressed cells was dose dependent when we varied the amount of added rCL-11 (0, 150, 300, and 600 $\mathrm{ng} / \mathrm{ml}$ ) (Figure 6, C and D). We concluded that both the concen- 


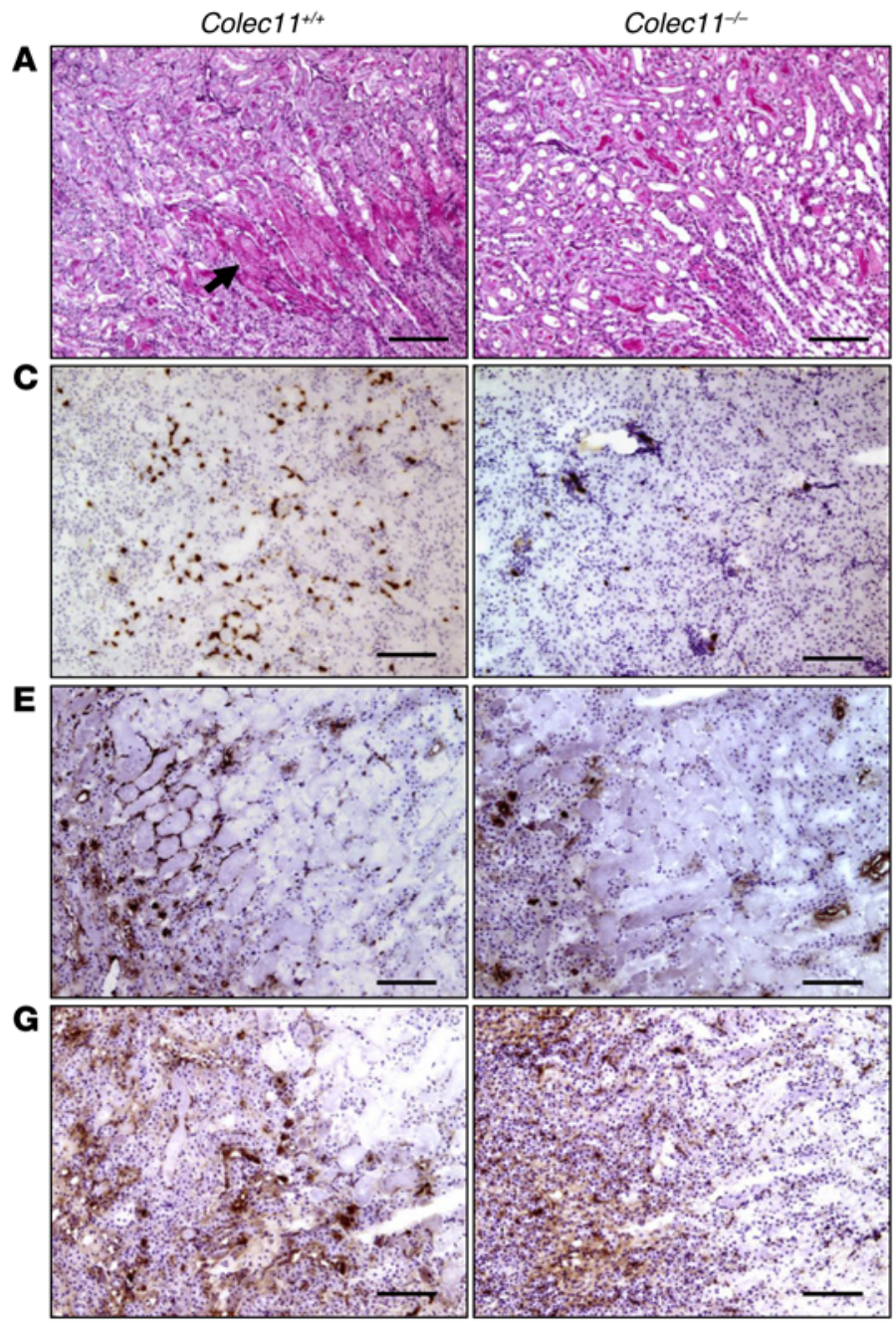

Ly-6B.2
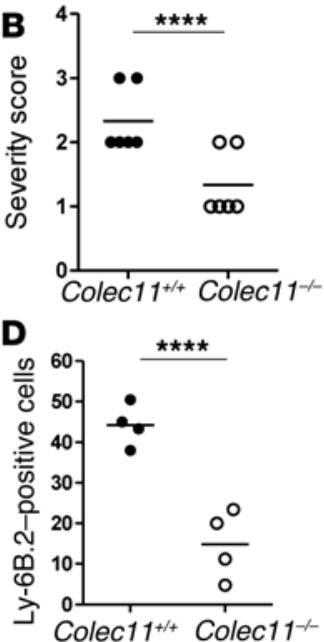

CD45

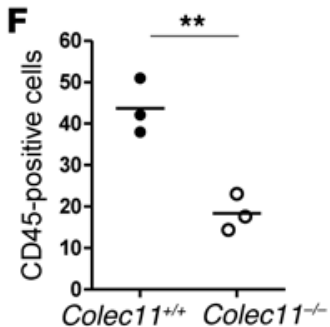

I
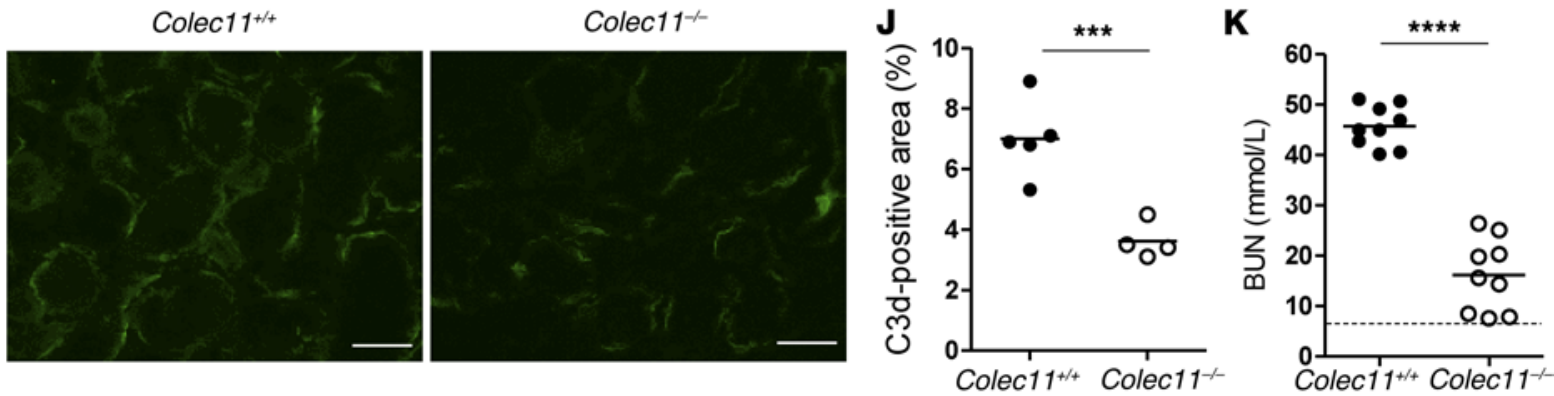

Figure 2. Impact of CL-11 on renal injury induced by $\mathbf{5 0}$ minutes of ischemia. Renal injury and complement activation in Colec $11^{+/+}$and Colec $11^{-/-}$mice following 50 minutes of ischemia and 24 hours of reperfusion. (A) Representative images of PAS staining of kidney sections from Colec $11^{+/+}$and Colec $11^{-/-}$ mice ( $n=10$ mice/group). Arrow indicates necrotic area of renal tubules seen in a longitudinal section at the junction of the renal cortex and medulla. Scale bars: $100 \mu \mathrm{m}$. (B) Severity scores for renal tubular injury in the mice represented in A. (C) Representative images of immunochemical staining of neutrophils in kidney sections from Colec $11^{+/+}$and Colec $11^{-/-}$mice $(n=4$ mice/group). (D) Quantification of neutrophils in kidney sections from the mice represented in C. (E) Representative images of immunochemical staining of leukocytes in kidney sections from Colec $11^{+/+}$and Colec $11^{-/-}$mice $(n=3$ mice $/$ group$)$. Scale bars: $100 \mu \mathrm{m}$. (F) Quantification of leukocytes in the kidney sections represented in E. (G) Representative images of immunochemical staining of macrophages in kidney sections from Colec $11^{+/+}$and Colec $11^{-/-}$mice $(n=3$ mice/group). Scale bars: $100 \mu \mathrm{m}$. (H) Quantification of macrophages in the kidney sections represented in G. (I) Representative images of immunofluorescence staining of C3d in kidney sections from Colec $11^{+/+}$and Colec $11^{-/-}$mice $(n=10$ mice/group). Scale bars: $25 \mu \mathrm{m}$. (J) Quantification of C3d in kidney sections from the mice represented in I. (K) BUN levels in Colec11+/+ and Colec $11^{-/-}$mice after reperfusion ( $n=10$ mice/group). Dashed line represents the BUN baseline in normal, nonmanipulated mice. (B, D, F, H, J, and $\mathbf{K})$ Each dot represents an individual mouse. ${ }^{*} P<0.05,{ }^{* *} P<0.01,{ }^{* *} P<0.005$, and ${ }^{* * * *} P<0.001$, by unpaired, 2 -tailed Student's $t$ test. 
tration of CL-11 in the extracellular space and the capacity of the stressed cell for CL-11 binding contributed to the complex pattern of interaction between CL-11 and the basal cell surface. Hypoxiaand hypothermia-induced patterns of CL-11 binding were indistinguishable in our experiments.

Human epithelial cells showed properties similar to those of mouse cells. We studied a transformed cell line derived from human RTECs (hRTECs), whose growth as suspension cells permitted the analysis of CL-11 binding by flow cytometry (22). We observed constitutive expression of CL-11 in permeabilized hRTECs (Supplemental Figure 7A). In addition, using nonpermeabilized cells to which rCL-11 had been added, we detected a dose-dependent increase in CL-11 binding on the cell (Supplemental Figure 7B). Thus, human cells can produce and bind CL-11, a finding that verifies the results with murine cells derived by primary culture and agrees with the reported expression of CL-11 in human renal tissue $(3,11)$.

$L$-fucose recognition by CL-11 on stressed cells. The specificity with which CL-11 can detect soluble or immobilized monosaccharide has been the subject of recent reports, which indicated that $\mathrm{L}$-fucose was the preferred ligand for both human and murine CL-11 $(3,11)$. L-fucose is a constituent of many $N$ - and $O$-linked glycoproteins and glycolipids that are produced by mammalian cells and can be present on the cell membrane or glycocalyx (23). Accordingly, we examined whether L-fucose could be the cellular ligand recognized by CL-11 in our experiments. We preincubated rCL-11 with soluble L-fucose, and in control studies, we used soluble D-galactose (which has minimal relevance to CL-11) (3). We then measured the ability of the monosaccharide-treated rCL-11 to bind to stressed Colec11 $11^{-/}$RTECs. While L-fucose treatment substantially prevented CL-11 binding to RTECs, D-galactose caused no major loss of CL-11 binding (Figure 7, A and B). This suggested that detection of L-fucose by the CRD on CL-11 was important for CL-11 binding to stressed renal tubule cells.

To verify that L-fucose on mammalian cells acts as a novel stress-associated binding ligand for CL-11, we treated stressed murine RTECs with fucosidase to specifically remove L-fucose from the cell surface (Figure 7C). We then examined the fucosedenuded cells (or control cells treated with galactosidase) for the ability to bind CL-11 after the addition of rCL-11. The fucosedenuded cells showed an extensive reduction of CL-11-binding capacity, while those pretreated with galactosidase showed no loss of CL-11 binding (Figure 7, D and E). Hence, the presentation of L-fucose on the stressed RTEC surface was required to induce significant CL-11 attachment, consistent with the colocalization of these 2 molecules in postischemic kidney (Figure 1E).

CL-11-ligand interaction triggers complement activation on stressed cells. We constructed an in vitro assay to test the hypothesis that complement activation on stressed renal tubule cells is initiated by CL-11 binding, which was suggested by the observed ability of C3d and CL-11 to localize at the site of tubule injury (Supplemental Figures 5 and 6). We exploited the recognized property of renal tubule cells to deposit complement from normal human serum, which contains the necessary complement components and activating factors to achieve cleavage of C3 into its active form $(24,25)$. We first confirmed this property in murine cells by showing that C3d was formed on stressed RTECs upon incubation with normal mouse serum (NMS) (Figure 8, A and B). We then examined the requirement for CL-11 and the associated lectin complement pathway enzyme MASP-2 using KO mouse sera that individually lack these specific components. Of the 3 MASPs known to be associated with CL-11, MASP-2 cleaves the complement component $\mathrm{C} 4$ and C4-bound $\mathrm{C} 2$ to form the central C3 convertase C4b2a (26). In addition, MASP-2-mediated cleavage of $\mathrm{C} 3$ can occur through a route that is independent of $\mathrm{C} 4$. The first report on the existence of this so-called $\mathrm{C} 4$ bypass was based on the analysis of C4-deficient mouse serum and $\mathrm{C} 4$-deficient human serum from a patient who was deficient in the human $\mathrm{C} 4$ genes $C 4 A$ and $C 4 B$ (26). In that report, reduced but clearly measurable lectin pathway-dependent cleavage of $\mathrm{C} 3$ was detected and could be inhibited by either adding MASP-2 inhibitory Abs or fluid-phase ligands to block lectin pathway-recognition subcomponents. C4-independent activation of $\mathrm{C} 3$ that is mediated by MASP-2 has also been described in several models of ischemia-reperfusion injury (IRI) including postischemic kidney injury $(26,27)$. Therefore, we examined the requirement for C4 in the present study as well as the need for CL-11 and MASP-2 in triggering complement activation on RTECs.

With either CL-11 or MASP-2 missing from the experimental protocol, we found that complement (C3d) deposition from the serum largely failed (Figure $8, \mathrm{~A}$ and $\mathrm{B}$ ). This indicated that both of these molecules were essential for enabling the conversion of C3 into its active form and promoting C3d formation on the injured cells, suggesting that the lectin complement activation pathway plays a key role in initiating complement activation. By contrast, complement deposition from C4-deficient mouse serum was unrestrained compared with that detected in NMS (Figure 8, A and B), suggesting that MASP-2 used the C4-bypass route to cleave $\mathrm{C} 3$ in this model $(26,27)$.

Having established that CL-11 was indispensable for triggering complement activation on the injured tubule cell, we asked whether it was the cell-bound or fluid-phase fraction of CL-11 that was essential. To resolve this question, we enzymatically removed L-fucose from the surface of stressed Colec11/- RTECs, thereby preventing the majority of CL-11 and C3d binding in NMS (Figure 8, C and D). By contrast, galactosidase-treated RTECs retained the ability to bind CL-11 and deposit C3d (Figure 8, C and D). The merged images for CL-11 and C3d showed strong colocalization of these 2 molecules (Figure 8C), indicating that complement was largely deposited at the site of CL-11 binding. In addition, we sought independent evidence that the membrane-bound fraction of CL-11 could trigger complement activation. Stressed Colec11/RTECs were coated with rCL-11 and washed to remove unbound rCL-11, followed by incubation of the washed cells with CL-11-deficient serum and detection of bound C3d. We found that substantial formation of C3d only occurred when the cells were pretreated with CL-11 (Figure 8, E and F).

This meant that both the cell-surface presentation of L-fucose and attachment of CL-11 were necessary to initiate MASP-2mediated cleavage of $\mathrm{C} 3$ in the context of renal tubule cell stress.

\section{Discussion}

While there is a broad understanding of the effector pathways that can mediate postischemic renal injury (28), the precise nature of 


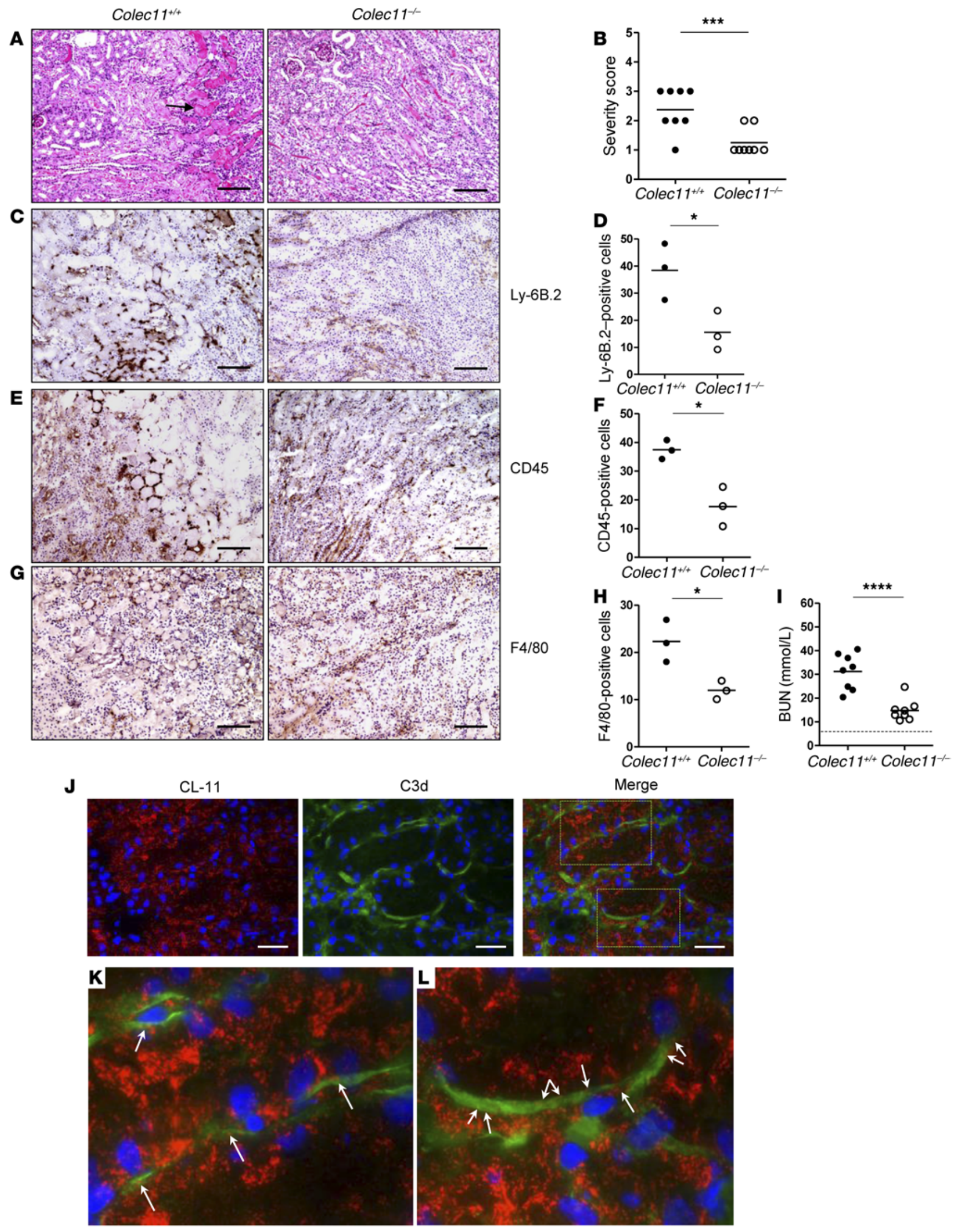


Figure 3. Impact of CL-11 on injury induced by milder ischemic insult. Renal injury is shown for Colec $11^{+/+}$and Colec $11^{-/-}$mice following 30 minutes of ischemia and 24 hours of reperfusion. (A) Representative images of PAS staining of kidney sections from Colec $11^{+/+}$and Colec $11^{-/-}$mice $(n=8$ mice $/ g r o u p)$. Arrow indicates necrotic renal tubules seen in a longitudinal section at the corticomedullary junction. Scale bars: $100 \mu \mathrm{m}$. (B) Severity scores for renal tubular injury in the mice represented in A. (C) Representative images of immunochemical staining of neutrophils in kidney sections from Colec $11^{+/+}$and Colec $11^{-1-}$ mice ( $n=3$ mice/group). Scale bars, $100 \mu \mathrm{m}$. (D) Quantification of neutrophils in the kidney sections represented in C. (E) Representative images of immunochemical staining of leukocytes in kidney sections from Colec $11^{+/+}$and Colec $11^{-/-}$mice $(n=3$ mice/group). Scale bars: $100 \mu \mathrm{m}$. (F) Quantification of leukocytes in the kidney sections represented in E. (G) Representative images of immunochemical staining of macrophages in kidney sections from Colec $11^{+/+}$and Colec $11^{-/-}$mice ( $n=3$ mice/group). Scale bars, $100 \mu \mathrm{m}$. (H) Quantification of macrophages in the kidney sections represented in panel G. (I) BUN levels in Colec $11^{+/+}$and Colec11 $1 /$ mice after reperfusion ( $n=8$ mice/group). Dashed line represents the BUN baseline in normal, nonmanipulated mice. (J) Representative immunofluorescence microscopic images of CL-11 (red) and C3d (green) and a merged image of CL-11 and C3d in ischemic kidney tissue. Scale bars: $25 \mu \mathrm{m}$. (K and $\mathbf{L}$ ) Enlarged images (original magnification, $\times \mathbf{4 0 0}$ ) of the merged image in J. Arrows indicate where the linear deposit of complement is dotted with CL-11 (orange) along the basolateral surface of the tubular epithelium, which is best appreciated at higher magnification. (B, D, F, $\mathbf{H}$, and I) Each dot represents an individual mouse. ${ }^{*} P<0.05$, ${ }^{* *} P<0.005$, and ${ }^{* * *} P<0.001$, by unpaired, 2-tailed Student's $t$ test.

stress-induced tissue changes and the associated molecules that initiate these responses are less certain. Here, we describe how CL-11 functions as a pattern-recognition molecule whose interaction with stress-inducible L-fucose on renal epithelial cells incites an inflammatory and tissue-destructive response. Two aspects of CL-11 function are at play: the detection of an aberrant pattern of carbohydrate that is linked to cell stress and the triggering of the complement cascade at the site of lectin attachment (Figure 9). Our results therefore identify CL-11 as a tissue-derived molecular trigger that initiates complement activation and local inflammatory responses relevant to acute kidney injury.

Our study defines L-fucose as a damage-associated ligand that is presented on epithelial cells following hypoxic or hypothermic conditions. L-fucose is a constituent of many glycans that are produced by mammalian cells and expressed on the cell membrane or glycocalyx coat (23). In ischemic tissue, we found an abnormal pattern of L-fucose expressed at the basolateral border of proximal tubules cells, in association with CL-11 and complement deposition. A display of the aberrant carbohydrate pattern was essential to fully induce CL-11 binding and complement activation on cultured renal tubule cells. We deduced that L-fucose was the primary ligand recognized by CL-11 on injured tubule cells, given the observation that blockade of the interaction of L-fucose with CL-11 prevented CL-11 binding and complement activation in our model. Mannose is another possible ligand recognized by CL-11 (3). However, our studies only detected mannose on the luminal surface, away from the basal surface targeted by complement in ischemic tissue (Supplemental Figure 8). The molecular identity of the fucose-bearing glycolipid or glycoprotein that was recognized by CL-11 on the tubule cells remains unclear. This will be an important factor in deciphering whether a change in orientation or exposure of the CL-11 ligand or an increase in ligand density and pattern of distribution are required to induce efficient binding of CL-11 on damaged cells. Work is underway to resolve this question.

Collectins such as MBL, SP-A , and SP-D are multifunctional molecules that can trigger several independently operating effector responses of the innate immune system $(6,7,9,10)$. The present study identifies complement as one such effector mechanism triggered by CL-11 on RTECs. Previous work has shown that CL-11 isolated from human serum is physically associated with the serine proteases MASP-1, MASP-2, and MASP-3, which are required for lectin complement pathway activation $(3,8)$. Our data suggest that the pathway of complement activation triggered by CL-11 on tubule cells used MASP-2, leading to cleavage of $\mathrm{C} 3$ via a C4-independent route. Complement activation that proceeds through MASP-2 but does not subsequently require $\mathrm{C} 4$ has been reported in several organ models of stress-induced injury including postischemic injury of the heart, intestine, and kidney $(26,27)$. Since CL-11 is expressed within these organs $(3,4,11)$, it is possible that CL-11-mediated lectin pathway activation underpins a common trigger mechanism. In contrast to CL-11, we found no evidence to support a significant role of MBL in our model, since complement deposition on cells largely failed when CL-11 was absent, even though MBL was present (in serum) in our experiments.

Our results do not exclude a secondary role for the alternative complement pathway, which in previous studies was shown to mediate ischemic acute renal failure in mice $(29,30)$. C3b formed by the lectin pathway could initiate alternative complement pathway activation by binding to native factor $\mathrm{B}$, thereby enabling factor $\mathrm{D}$ to cleave $\mathrm{C} 3 \mathrm{bB}$ and form the alternative pathway $\mathrm{C} 3$ convertase $\mathrm{C} 3 \mathrm{bBb}$ (31). Additionally, the lectin pathway proteases MASP-1 and MASP-2 could synergistically promote alternative pathway activation by converting pro-factor $\mathrm{D}$, the zymogen form of factor D, into its enzymatically active form $(32,33)$. In our study, it is therefore feasible that alternative pathway activation could have substantially increased the amount of complement deposition following initiation of the lectin pathway through CL-11. This may explain the disproportionate quantity of C3d relative to CL-11 we detected along the lateral border of the renal tubule in ischemic studies. Moreover, "tick-over" activity of the alternative pathway could have accounted for the residual amount of C3d deposited when CL-11 was absent from the experiment. Future studies in mice with combined deficiency of the lectin and alternative pathways will be useful in addressing these subsidiary mechanisms and their dependence on CL-11.

CL-11 and CL-10 are detected in normal human serum as CL-11 and CL-10 heterotrimers alongside CL-11 and CL-10 homotrimers (34). It was reported that interchain disulphide bonds held together the CL-10 and CL-11 single chains, implying that such heterotrimers can only form within a cell that synthesized both components (34). So far, the only known cell in which CL-11 and CL-10 are coexpressed is the hepatocyte. We therefore did not examine CL-10 in our study, since the published results claim that only CL-11 is expressed by renal tissue. The specific physiological role of the CL-10 and CL-11 heterotrimers is unknown. However, from our perspective, the absence of CL-11 in the gene-targeted mouse strain that we analyzed gave us the opportunity to demon- 


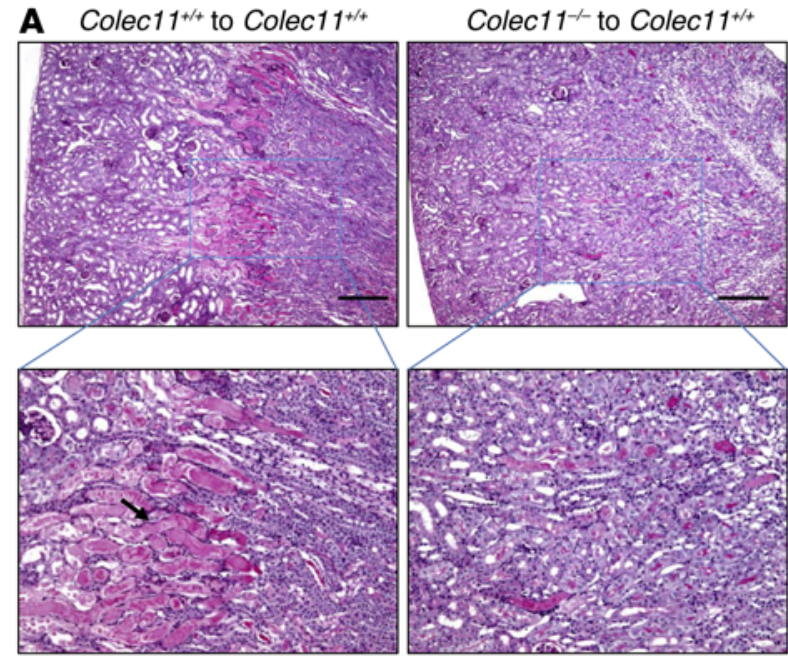

Figure 4. Contribution of kidney-derived CL-11 in post-transplantation ischemic injury. Quantification of histological injury and/or renal dysfunction is shown for Colec $11^{1 /+}$ mice transplanted with kidneys from Colec $11^{+/+}$or Colec $11^{-/-}$littermates. The donor organ was kept on ice for 25 minutes prior to implantation. (A) Histology with PAS staining 24 hours after transplantation representing the peak of injury ( $n=2$ mice/group). Enlarged panel (original magnification, $\times 200$ ) with an arrow illustrates necrotic renal tubules seen in a longitudinal section. Scale bars: $100 \mu \mathrm{m}$. (B) Severity scores for the renal tubular injury represented in A. (C) BUN measurement in the recovery phase on day 7 after transplantation for recipients of $\mathrm{Colec}_{11^{+/+}}$or $\mathrm{Colec} 11^{-/-}$donor kidneys, following the remaining native nephrectomy on day 6 ( $n=6$ mice/group). Dashed line represents the BUN baseline in normal, nonmanipulated mice. (D) Severity scores for the renal tubular injury in mice represented in $\mathbf{C}$. ${ }^{*} P<0.05$ and ${ }^{*} P<0.01$, by unpaired, 2 -tailed Student's test.

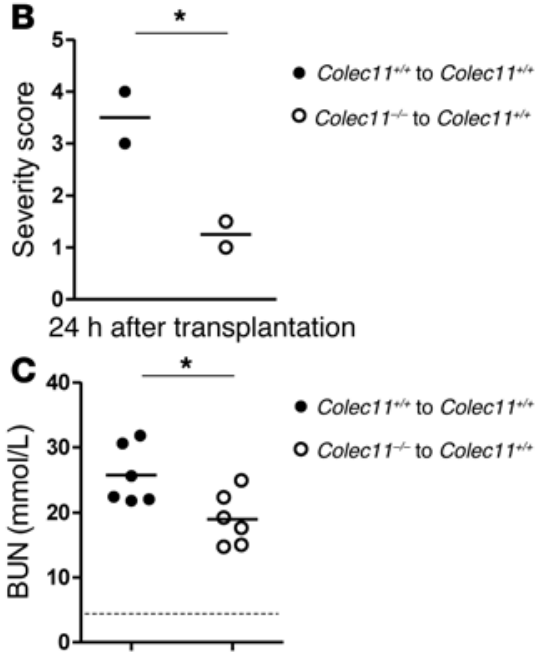

Day 7 after transplantation
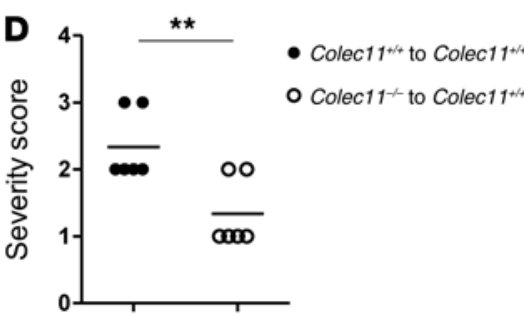

Day 7 after transplantation strate that it is the locally produced CL-11 in the kidney that triggers renal ischemic injury in a MASP-2-dependent fashion, since our transplantation experiments clearly show that the implantation of Colec11 $1 /-$ kidneys into CL-11-sufficient (and CL-10-sufficient) WT mice achieves a significant degree of protection against ischemia-reperfusion damage. It is therefore not plasma-derived CL-11 that triggers the lectin pathway within the ischemic kidney. Furthermore, it is clear from our in vitro studies using recombinant CL-11 added to cultured renal tubule cells that CL-11 reacts directly with the L-fucose presented by target cells.

Susceptibility of renal tubule cells to complement-mediated injury is associated with a number of factors. These epithelial cells express the central complement component C3 $(18,35,36)$, which, when cleaved, leads to formation of the membrane attack complex C5b-9 and release of C5a $(18,20)$. Both of these complement effectors stimulate proinflammatory responses in epithelial cells and infiltrating leucocytes and can mediate cell death $(17,37,38)$. In addition, renal tubule cells show relatively weak expression of cytoprotective complement regulators that inhibit cleavage of $\mathrm{C} 3$ into its active state, which means the cells have a low injury threshold (39). Here, we show that, in addition to these factors, renal tubule cells can release CL-11, which, in the presence of cell stress, can initiate complement activation on the tubule surface. Since cultured cells deposited minimal complement in the absence of CL-11, this indicates that the lectin pathway activation triggered by CL-11 was the primary route of complement activation on these cells. The cell-autonomous action of CL-11 reported here in the context of epithelial cell stress is distinct from the potential action of other systemic collectins such as MBL, whose central produc- tion (by hepatocytes) and formation of large circulating oligomers (200-600 kDa) may prevent MBL from having a significant biological role at some extravascular sites (6).

Our findings leave little doubt that the proximal tubule cell was the source of the CL-11 involved in the mediation of postischemic renal injury. The transplantation studies identified an important contribution of kidney-derived CL-11, while segment-specific mapping studies showed that only proximal tubule cells expressed the combination of CL-11 and L-fucose that was required to trigger lectin complement pathway activation. We do not claim that CL-11 interaction with its major ligand L-fucose was the sole mechanism of complement activation on hypoxia-sensitive tubules, since background levels of complement activation occurred in the absence of CL-11 and minor complement deposition affected the distal tubules independently of L-fucose. Nonetheless, our results establish the idea that complement deposition was predominantly associated with the proximal tubule, where the molecular components required for lectin pathway activation were coexpressed. Tubule segment-specific deletion of CL-11 could provide a future means to corroborate these findings and possibly discover other functions of CL-11 in different segments of the tubule.

In conclusion, $\mathrm{CL}-11$ is a soluble $\mathrm{C}$-type lectin with wide tissue distribution and can be produced locally in renal epithelial cells to initiate complement activation following cell-autonomous interaction with stress-induced ligand (L-fucose). The broad expression of CL-11 $(3,4,11)$ and its putative ligands $(3,11)$ makes it possible that CL-11 operates on a wider scale, promoting inflammation and immunity in other organs and conditions. Furthermore, the simplicity with which soluble monosaccharide inhib- 
A

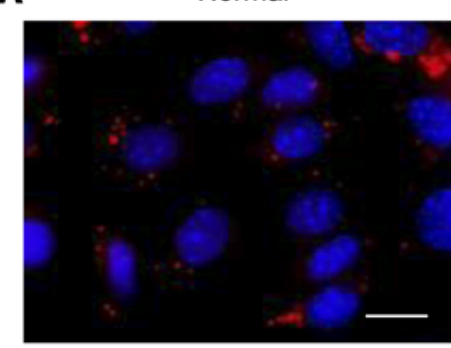

c

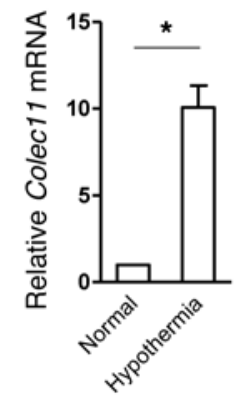

E

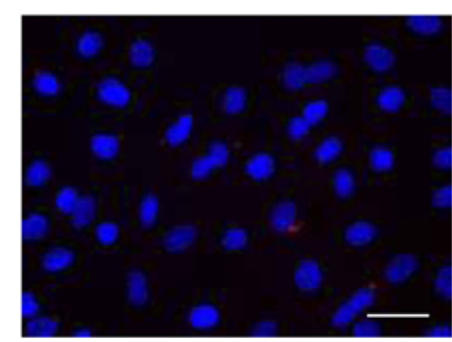

Hypoxia

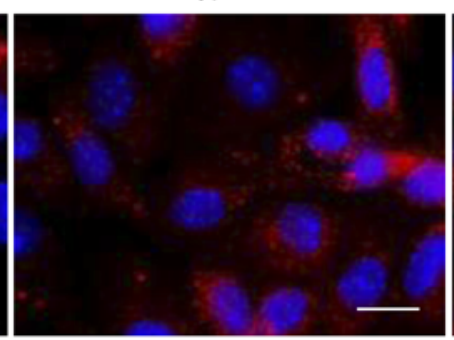

D

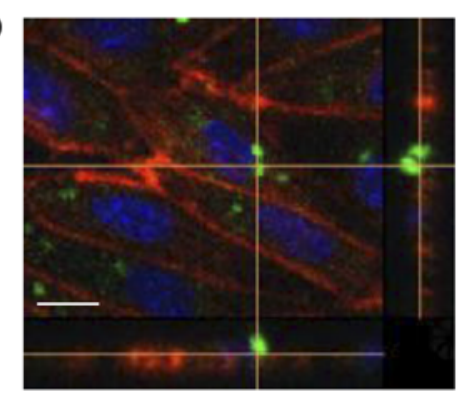

Nonstressed sup

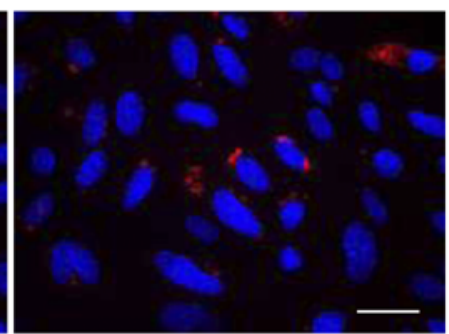

Hypothermia

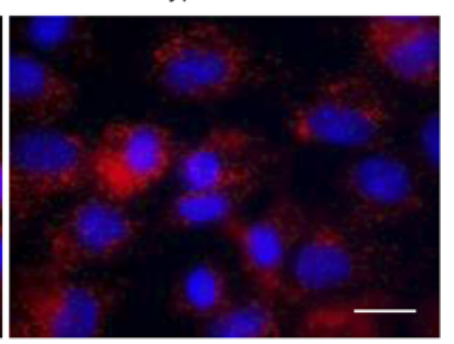

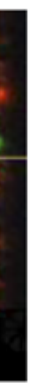

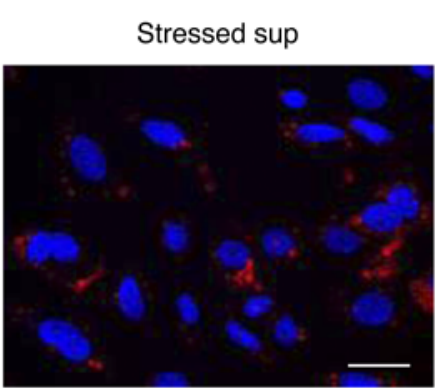

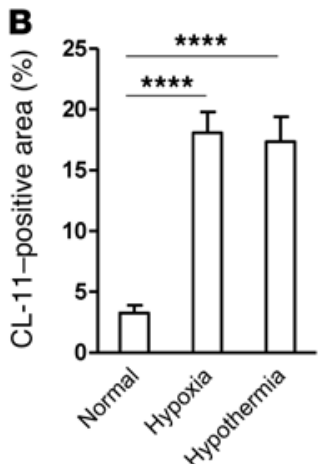

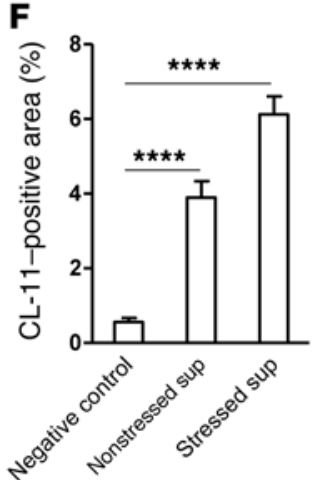

Figure 5. Renal tubule cell stress mediates CL-11 expression. (A) Representative immunofluorescence images of intracellular CL-11 in nonstressed and hypoxia-stressed RTECs or in hypothermia-stressed Colec11+/+ RTECs (permeabilized). CL-11 (red) and nuclei (blue) are shown. Scale bars: $10 \mu$ m. (B) Quantification of intracellular CL-11 in the RTECs shown in A. Data shown are from 6 individual images and representative of 4 independent experiments. (C) Relative Colec 11 mRNA levels in nonstressed and hypothermia-stressed Colec $11^{+/+}$RTECs, as determined by qRT-PCR ( $n=3$ ). Data are representative of 2 experiments. ${ }^{*} P<0.05$, by unpaired, 2-tailed Student's $t$ test. (D) Confocal microscopic image of CL-11 in hypothermia-stressed Colec11/++ RTECs (nonpermeabilized). CL-11 (green), F-actin (red), and nuclei (blue) are shown. Views in the bottom and side panels show that CL- 11 was present at the basal cell surface. (E) Representative immunofluorescence images of bound CL-11 in Colec11-/- RTECs that had been hypoxia stressed and then incubated with supernatants from nonstressed (Nonstressed sup) or hypoxia-stressed (Stressed sup) Colec $11^{+/+}$RTEC cultures or nonstressed Colec11 ${ }^{-/-}$RTEC cultures (negative control), demonstrating that the supernatants from Colec11+/+ RTEC cultures had CL-11-binding activity. Scale bars: $25 \mu \mathrm{m}$. (F) Quantification of bound CL-11 in the RTECs represented in E. Data shown are from 7 individual images and representative of 2 independent experiments. (B and F) ${ }^{* * * *} P<0.001$, by 1 -way ANOVA.

ited CL-11 binding to targeted epithelial cells in our study could suggest a physiological control mechanism that merits further exploration and exploitation.

\section{Methods}

We used the following reagents and materials: isofluorane (Abbott); microaneurysm clamps (Codman); urease kit (BUN Infinity); and Vivaspin ultrafiltration spin columns (Fisher Scientific); polyclonal goat anti-human CL-11 Ab (S14; Santa Cruz Biotechnology Inc.); polyclonal rabbit anti-human $\mathrm{C} 3 \mathrm{~d} \mathrm{Ab}$ (A0063; Dako) and HRP-conjugated rabbit anti-rat Ig Ab (P0450; Dako); rat anti-mouse Ly-6B.2 mAb (clone 7/4; AbD Serotec); donkey anti-goat Alexa Fluor 568 Ab (A11057; Invitrogen); FITC-conjugated goat anti-rabbit IgG Ab (111-095-144; Stratech Scientific); DAPI (Life Sciences); fluorescein-conjugated lotus tetragonolobus lectin (LTL) (Vector Laboratories); collagenase
II (Lorne Laboratories); Texas red phalloidin (Molecular Probes); cell culture medium (DMEM/F12), FCS, and ITS (mix of insulin, transferrin, and selenium) (Invitrogen); hydrocortisone, triiodothyronine, and enzymes (i.e., L-fucose, D-galactose, L-fucosidase, and $\beta$-gal) (SigmaAldrich); and rCL-11 (Stratech Scientific).

Animals. WT and KO mice with deficiencies of CL-11, MASP-2, or C4 were on a C57BL/6 background $(27,40,41)$. Homozygous CL-11$\mathrm{KO}\left(\mathrm{Colec}_{\left.11^{-/}\right)}\right)$mice were purchased from Mutant Mouse Resource and Research Centers (MMRRC) (UC Davis, Davis California, USA) (40). WT littermates $\left(\right.$ Colec $\left.11^{++}\right)$were used as controls. Male mice $(\sim 22 \mathrm{~g} \mathrm{BW}$; 6-8 weeks of age) were used in all experiments. All mice were maintained in specific pathogen-free conditions.

Induction of renal IRI. Renal IRI was induced as we described previously (20). Briefly, mice were anesthetized by inhalation of isofluorane and kept warm on a heated pad. An abdominal incision was per- 

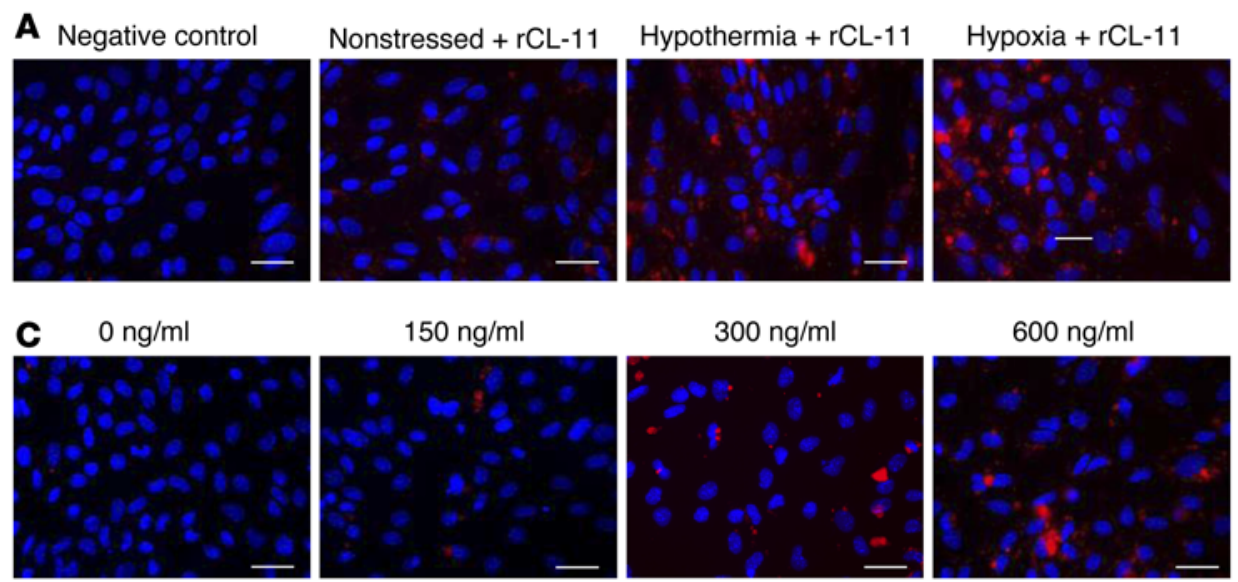

Figure 6. Renal tubule cell stress mediates CL-11 binding. Binding of exogenous CL-11 detected by immunofluorescence staining after incubation of $\mathrm{rCL}-11$ with Colec11/- RTECs is shown. (A) Representative immunofluorescence images of bound $\mathrm{rCL}-11$ in nonstressed and stressed (i.e., hypothermia or hypoxia) Colec $11^{-1-}$ RTECs after incubation with rCL-11, showing a clumped CL-11-binding pattern induced by cell stress. The negative control consisted of stressed Colec $11^{-1-}$ RTECs that had not been incubated with rCL-11. Scale bars: $25 \mu \mathrm{m}$. (B) Quantification of bound CL-11 corresponding to the RTECs shown in A. Data shown are from 10 individual images and representative of 3 independent experiments. ${ }^{* * *} P<0.001$, by 1 -way ANOVA. (C) Dose titration of $\mathrm{rCL}-11$ binding to stressed RTECs. Representative images show the different CL-11 concentrations. Data shown are from 6 to 8 individual images and representative of 2 independent experiments. Scale bars: $25 \mu \mathrm{m}$. (D) Quantification of bound CL-11 corresponding to the RTECs represented in C. Data were analyzed by 1-way ANOVA ( $P$ value is indicated in the graph).
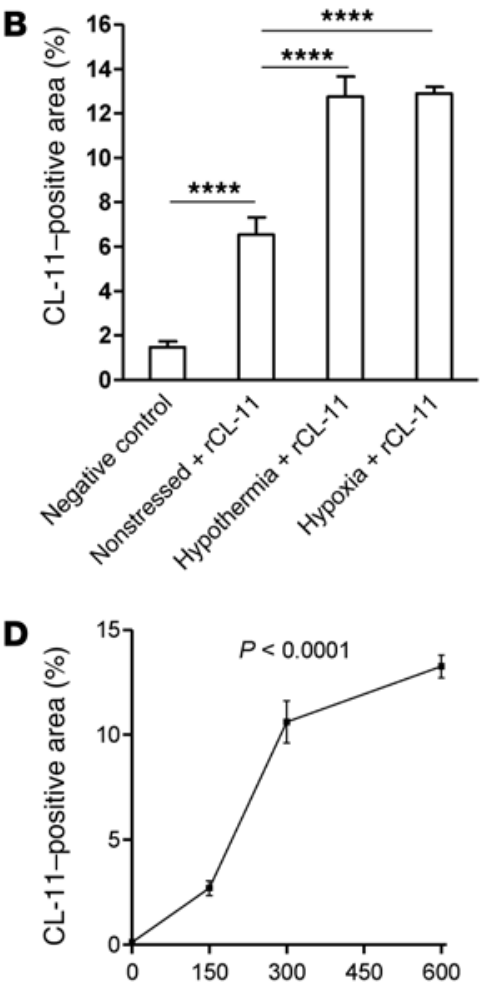

Concentration of rCL-11 [ng/ml] formed, and renal arteries and veins were isolated and then bilaterally occluded for 50 minutes or 30 minutes using microaneurysm clamps that provide the correct amount of pressure to provide complete occlusion without damaging the vessel walls. Following removal of the clamps, $0.5 \mathrm{ml}$ warm saline was placed in the abdomen, the incision was sutured, and the animals were placed in a warm box to recover. Mice were killed at either 6 or 24 hours or 0.5 hours after removal of the clamps. Blood samples were taken for the assessment of renal function, and kidneys were harvested for histopathology. The moderate severity of injury achieved with 50 minutes of occlusion is associated with a survival rate of $90 \%$ or higher at day 7 after ischemia.

Mouse kidney transplantation. Mice were anesthetized by inhalation of oxygen and isofluorane (Abbott Laboratories) and kept warm using a heated mat. The donor kidney was surgically removed along with an arterial patch. The renal vein was cut close to the inferior vena cava, and the ureter was isolated and cut close to the bladder. The kidney was then placed in ice-cold saline for 25 minutes, during which time the recipient was anesthetized. The recipient native right kidney was removed, and microaneurysm clamps (the same type used in the native model of ischemia) were placed to occlude the aorta and inferior vena cava (IVC). Incisions were made in the aorta and IVC, to which donor artery and vein were anastomosed. The period of warm ischemia during the formation of this anastomosis lasted for 30 minutes. The clamps were then removed, and the kidney was observed to ensure revascularization. The ureter was connected to the bladder, and $0.5 \mathrm{ml}$ warm saline was placed in the body cavity. The abdomen was closed and the animal returned for recovery to a heated chamber for 24 hours. The remaining native kidney was excised on day 6 after transplantation, and the animal was killed after a further 24 hours. Blood and kidney were collected for assess- ment of renal function and tissue damage, respectively. In separate experiments, recipients were killed 24 hours after successful transplantation so that the peak injury could be assessed histologically. This procedure is associated with a mortality rate of $10 \%$ or lower.

Assessment of renal function. Following the preparation of serum from collected blood, BUN was assessed using a standard urease kit (BUN Infinity) that measured coupled enzyme reactions involving urease and glutamate dehydrogenase.

Assessment of renal histopathology. Kidneys were fixed in a solution of $4 \%$ formalin in PBS for 24 hours and embedded in paraffin. Paraffin sections $(2 \mu \mathrm{m})$ were stained with periodic acid-Schiff (PAS). The severity of tubular injury at the corticomedullary junction was graded in a blinded fashion by 2 experimenters who used the following 5-point scale: 0, normal kidney; 1, less than 10\% necrosis; 2, 10\%-25\% necrosis; $3,25 \%-75 \%$ necrosis; and 4 , more than $75 \%$ necrosis, as we described previously (20).

Immunochemical staining of kidney tissues. Frozen sections $(4 \mu \mathrm{m})$ of OCT-embedded kidneys were air dried and then acetone fixed. Sections were incubated with polyclonal goat anti-human CL-11 Ab (1:100; Santa Cruz Biotechnology Inc.), which cross-reacts with mouse CL-11; polyclonal rabbit anti-human C3d Ab (1:200; Dako) or rat anti-mouse Ly-6B.2 mAb (1:100; Serotec); rat anti-mouse CD 45 Ab (clone 30-F11; 1:100; Caltag); and rat anti-F4/80 Ab (clone BM8; 1:100; Abcam), followed by secondary donkey anti-goat Alexa Fluor 568 (Life Technologies); FITC-conjugated goat anti-rabbit IgG Ab (1:200; Stratech Scientific); and HRP-conjugated rabbit anti-rat (for Ly-6B.2, CD45, and F4/80; 1:100; Dako), respectively. In all fluorescence immunohistochemical assays, cell nuclei were stained with DAPI (1:10,000). Markers for distal tubules (renal thiazide-sensitive $\mathrm{NaCl}$ cotransporter [NCC]) were detected with polyclonal rabbit anti-human NCC 
A

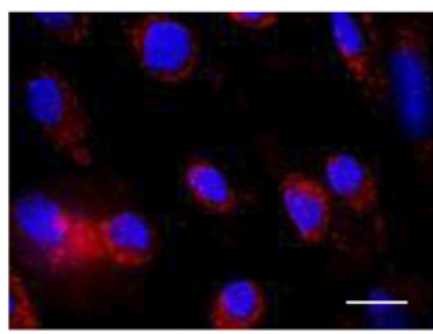

L-fucose

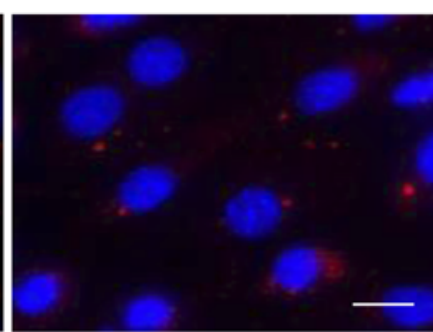

D-galactose

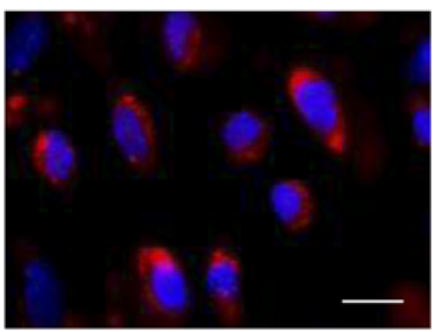

B

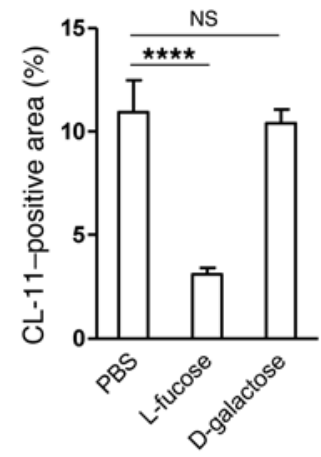

c

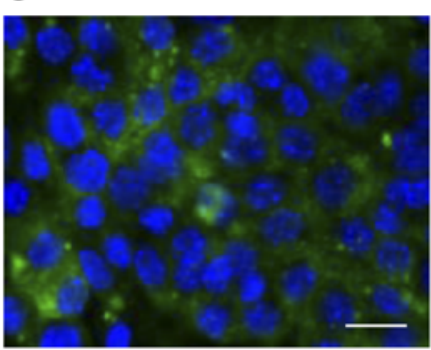

D

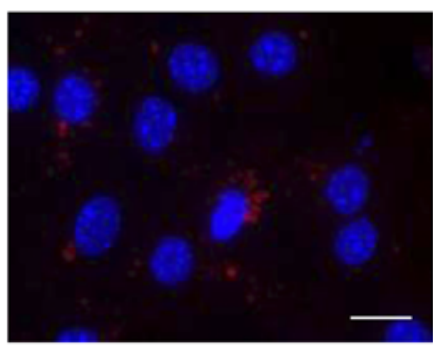

Fucosidase

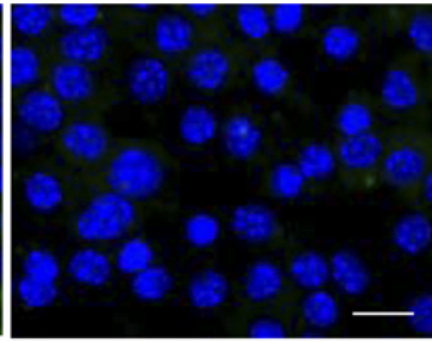

Fucosidase

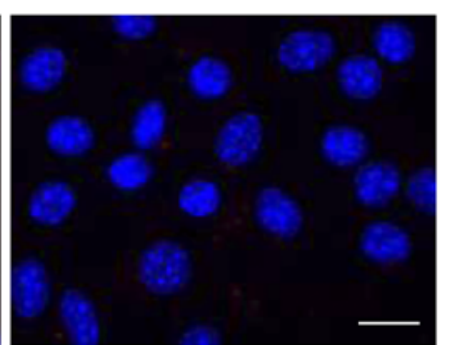

E

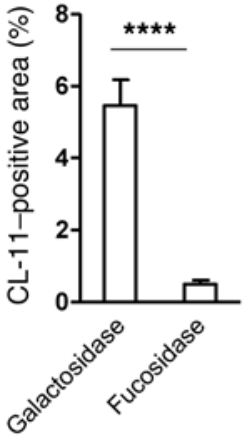

Figure 7. Sugar specificity of CL-11 binding to stressed renal tubule cells. (A) Representative immunofluorescence images of bound rCL-11 in hypothermia-stressed Colec11 ${ }^{-/-}$RTECs following incubation of the cells with rCL-11, which had been pretreated with PBS, L-fucose, or D-galactose, demonstrating that L-fucose-treated rCL-11 had reduced binding to the cells compared with that seen with PBS or D-galactose treatment. Scale bars: $10 \mu \mathrm{m}$. (B) Quantification of bound rCL-11 in the RTECs shown in $\mathbf{A}$. Data shown are from 10 individual images and representative of 3 independent experiments. ${ }^{* * *} P<0.001$, by 1-way ANOVA. (C) Representative fluorescence images of L-fucose (green) in hypothermia-stressed Colec11 ${ }^{-/-}$RTECs following pretreatment of the cells with $\alpha$-L-fucosidase or $\beta$-gal, as detected by the fluorescein-conjugated plant lectin LTL, demonstrating that fucosidase treatment reduced L-fucose residues on the cells. Scale bars: $12.5 \mu \mathrm{m}$. (D) Representative immunofluorescence images of bound rCL-11 (red) in the cells shown in C, which had been further incubated with rCL-11, demonstrating that fucosidase treatment reduced rCL-11 binding to the cells. Scale bars: $12.5 \mu \mathrm{m}$. (E) Quantification of bound rCL-11 in the RTECs shown in D. Data shown are from 6 to 10 individual images and representative of 2 independent experiments. ${ }^{* * *} P<0.001$, by unpaired 2-tailed Student's $t$ test.

$\mathrm{Ab}$ (1:300; EMD Millipore), followed by secondary donkey anti-rabbit $\mathrm{Cy} 5 \mathrm{Ab}$ (1:200). The detection of L-fucose was achieved using fluorescein-conjugated LTL (1:40). Following immunochemical staining of neutrophils, infiltration was quantified by counting positively stained cells at the corticomedullary junction area, at a magnification of $\times 200$, and expressed as the number of neutrophils per field.

RTEC cultures. Primary RTEC cultures grown as a monolayer were prepared from kidneys of Colec $11^{+/+}$or Colec11 ${ }^{-/}$mice as described previously (42). In brief, minced cortex was digested with $0.1 \%$ collagenase II and passed through a $40-\mu \mathrm{m}$ nylon sieve. The cells and tubules were collected and cultured in DMEM/F12 medium containing $2 \% \mathrm{FCS}$, insulin ( $5 \mu \mathrm{g} / \mathrm{ml})$, transferrin ( $5 \mu \mathrm{g} / \mathrm{ml})$, selenium $(5 \mathrm{ng} / \mathrm{ml})$, hydrocortisone (40 $\mathrm{ng} / \mathrm{ml})$, and triiodothyronine $\left(10^{-12} \mathrm{M}\right)$. Nonpassaged cells cultured for 7 to 9 days were used for the experiments described below.

Induction of RTEC stress. Thermal stress was induced by placing the RTECs at $4^{\circ} \mathrm{C}$ for 18 hours in full culture medium without FCS, followed by a period of recovery, in which cells were incubated at $37^{\circ} \mathrm{C}$ with $5 \% \mathrm{CO}_{2}$ for 2 hours. Hypoxia stress was induced by culturing RTECs under hypoxic conditions $\left(5 \% \mathrm{CO}_{2}, 1 \% \mathrm{O}_{2}\right.$ and $\left.94 \% \mathrm{~N}_{2}\right)$ in a gas chamber (Billups-Rothenberg) for 24 hours, followed by reoxygenation $\left(21 \% \mathrm{O}_{2}\right.$ and $\left.5 \% \mathrm{CO}_{2}\right)$ for 24 hours.

Detection of endogenous CL-11 in cultured RTECs. To detect the synthesis of CL-11 by RTECs, stressed or nonstressed RTECs were fixed in $4 \%$ paraformaldehyde for 10 minutes at room temperature and permeabilized with $0.2 \%$ Triton $\mathrm{X}-100$ for 3 minutes. Immunofluorescence for CL-11 was then performed as described earlier. To detect the secretion of CL-11 from RTECs, supernatants were collected from Colec11 ${ }^{+/+}$or Colec11 $1^{-/-}$(negative control) RTEC cultures and concentrated 20-fold using Vivaspin ultrafiltration spin columns. Supernatants were then added to Colec11 $1 /$ RTEC monolayers (which were hypoxia stressed) and incubated for 1 hour. The cells were then fixed, but not permeabilized, and subjected to immunofluorescence for CL-11.

Detection of exogenous CL-11 binding to cultured RTECs. RTECs cultured from Colec11 ${ }^{--}$kidneys were subjected to thermal or metabolic 

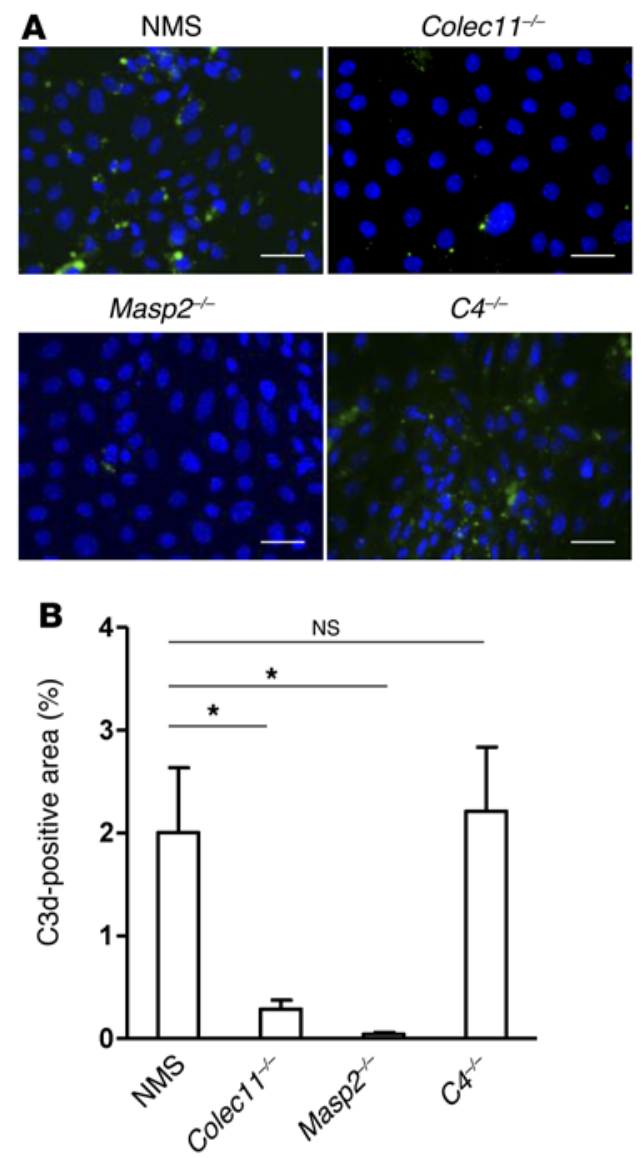

$\mathbf{E}$

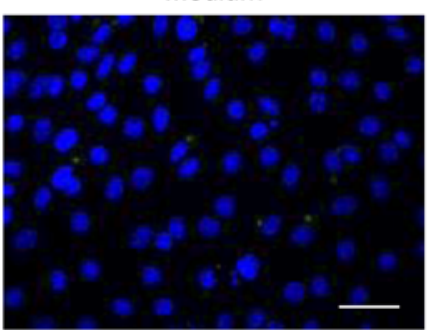

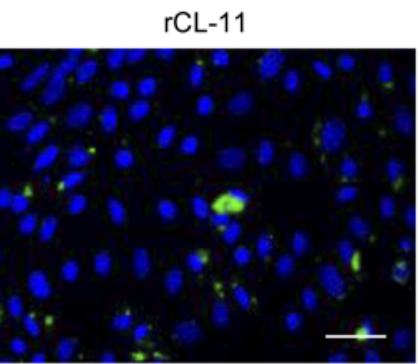
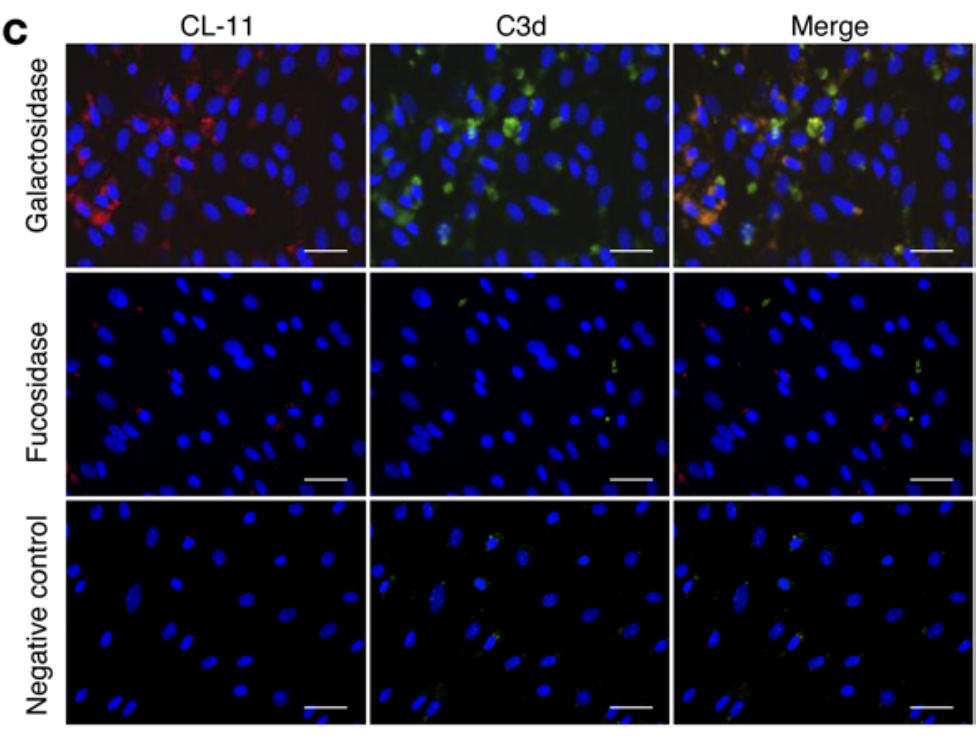

D
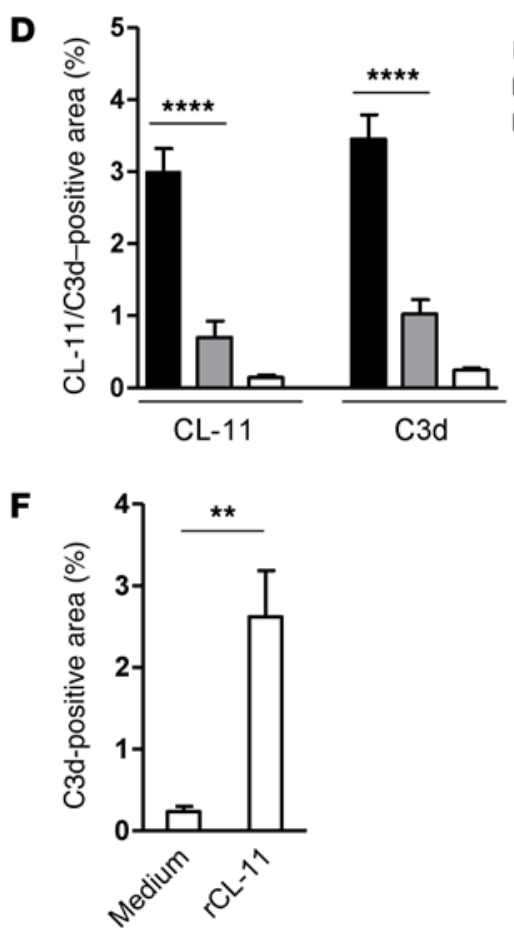

Figure 8. Requirement of complement subcomponents for complement activation on tubule cells. (A) Representative immunofluorescence images of C3d deposits on hypothermia-stressed Colec $11^{-/-}$RTECs following incubation with $10 \%$ fresh mouse serum from normal (NMS), Colec $11^{-1-}$, Masp2 $2^{-1 \text {, }}$ or $\left[4^{-/-}\right.$mice, demonstrating C3d deposition following incubation with NMS and reduced deposition with Colec $11^{-/-}$or Masp2 $2^{-/-}$serum; in contrast, C3d deposition was not reduced following incubation with $\mathrm{C4}^{-/-}$serum. Scale bars: $25 \mu \mathrm{m}$. (B) Quantification of C3d deposits on the RTECs shown in A. Data shown are from 10 individual images for each of 4 independent experiments. Percentage of C3d-positive binding to Colec $11^{1-}$ RTECs was determined from 5 to 7 individual fields, representative of 4 independent experiments, except for incubation with $\left[4^{-/-}\right.$serum, which was performed in 2 of those experiments. ${ }^{*} P<0.05$, by 1 -way ANOVA. (C) Representative fluorescence images of CL-11 (red), C3d (green), and a merged image of CL-11 and C3d in Colec $11^{-1-}$ RTECs that had been hypothermia stressed, pretreated with $\alpha$-L-fucosidase or $\beta$-gal, and then incubated with NMS, demonstrating that CL-11 binding and C3d deposition were reduced by fucosidase treatment. A negative control of $\alpha$-L-fucosidase-treated Colec11 ${ }^{-/-}$RTECs, which had been incubated with Colec11//- serum, is also shown. Scale bars: $25 \mu \mathrm{m}$. (D) Quantification of CL-11 binding and C3d deposition in the RTECs shown in C. Data shown are from 6 to 8 individual images and representative of 3 independent experiments. ${ }^{* * *} P<0.001$, by 2 -way ANOVA. (E) Typical staining pattern for $\mathrm{C} 3 \mathrm{~d}$ on stressed Colec11 ${ }^{-1-}$ RTECs that had been incubated with medium alone or medium containing rCL-11 and then washed and subsequently incubated with Colec11 $1 /-$ serum. (F) Quantification of C3d deposition in the RTECs shown in E. Data are from 6 individual images for each of 2 independent experiments. ${ }^{*} P<0.01$, by unpaired, 2 -tailed Student's $t$ test. 


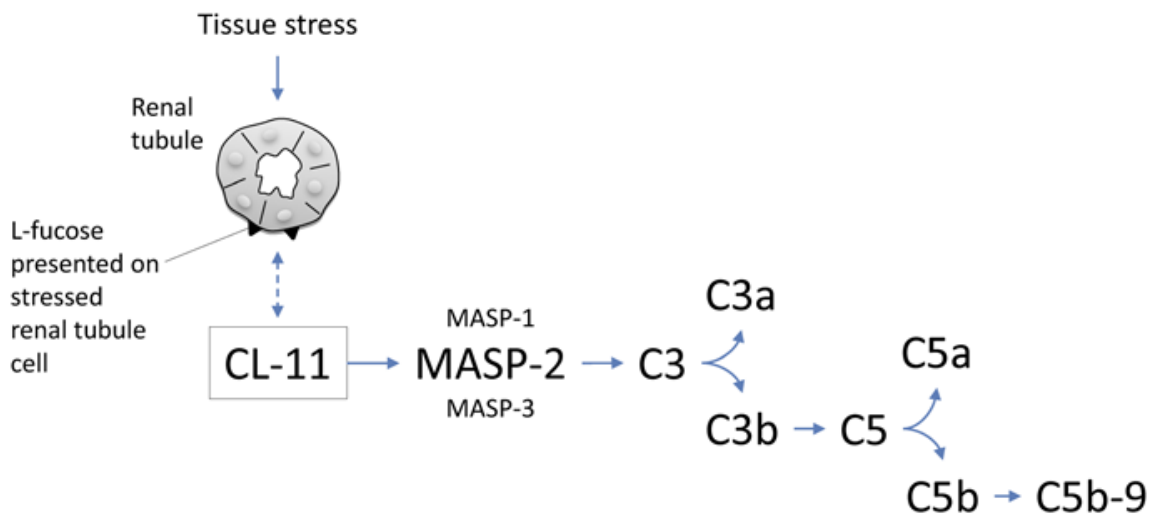

Figure 9. Proposed scheme in which CL-11 detects stress-associated L-fucose on renal tubule cells and initiates complement activation via the lectin pathway. Renal cell stress increases the concentration of CL-11 in the extracellular space and leads to cell-autonomous binding through abnormal presentation of L-fucose on the cell surface. High-density binding of CL-11 in the presence of MASP-2 results in the cleavage of $\mathrm{C} 3$. Complement effectors generated downstream of $\mathrm{C} 3$ are known to contribute to inflammatory cell infiltration and tubule cell death through receptor-mediated stimulation by $C 5 \mathrm{a}$ and membrane pore formation of $\mathrm{C} 5 \mathrm{~b}-\mathrm{9}$. This model depicts the lectin pathway triggered by CL-11 as the primary driver of complement activation in postischemic acute kidney injury.

immunostained CL-11, L-fucose, NCC, and C3d in normal and ischemic mouse kidney tissue. It was also used to determine the cellular location of deposited CL-11 on stressed cultured Colec11/- RTECs in relation to F-actin.

Semiquantitative real-time RT-PCR. Total RNA extraction from kidney tissue or cells, reverse transcription, cDNA synthesis, and PCR were performed as previously described (18). Relative gene expression was analyzed using the $2^{-\Delta \Delta C t}$ method (43) and expressed as $2^{-\Delta \Delta}(\mathrm{Ct})$, where $\mathrm{Ct}$ is the cycle threshold; $\Delta \Delta$ $(\mathrm{Ct})=$ test samples $\Delta(\mathrm{Ct})$ - control samples $\Delta$ (Ct); and $\Delta(\mathrm{Ct})=$ test gene $(\mathrm{Ct})-18 \mathrm{~s}(\mathrm{Ct})$. RNA extracted from normal, nonmanipulated kidney and nonstressed, cultured RTECs served as controls. The following primers were used for RT-PCR: murine Colec11: forward, 5'-TTCGCCTAGTGTGCATCCAG-3' and reverse, 5'-GCGCCAATGGGACCAATTTT-3'; 18s: forward, 5'-ATCCCTGAGAAGTTCCAGCA-3' and reverse, 5'-CCTCTTGGTGAGGTC-

stress as described above. Following the recovery period, cells were incubated with serum-free full culture medium supplemented with varying concentrations of rCL- 11 at $37^{\circ} \mathrm{C}$ for 1 hour. Cells were then thoroughly washed and fixed with $4 \%$ paraformaldehyde (but not permeabilized) and subjected to immunofluorescence for CL-11.

Evaluation of CL-11 binding to RTECs through L-fucose. Two different approaches were used: (a) assessment of the inhibition of CL-11 binding to RTECs by pretreatment CL-11 with L-fucose; and (b) assessment of the inhibition of CL-11 binding to RTECs by removal of L-fucose from the surface of RTECs. For the first approach, rCL-11 was incubated with either L-fucose $(2 \mathrm{mg} / \mathrm{ml})$ or D-galactose $(2 \mathrm{mg}$ / $\mathrm{ml}$ ) for 1 hour at room temperature and then applied in the assay of binding CL-11 to RTECs as described in the above section. For the second approach, Colec11 $1^{-/}$RTECs were treated with the enzyme $\alpha$-L-fucosidase or control enzyme $\beta$-gal $(0.1 \mathrm{U} / \mathrm{ml})$ or buffer alone for 1 hour at $37^{\circ} \mathrm{C}$. After treatment, the RTECs were used for the assay of bound CL-11 as described in the above section.

Detection of CL-11 and C3d deposition on cultured RTECs. RTECs cultured from Colec11 $1 /-$ kidneys were incubated with $10 \%$ serum from normal mice or Colec11 $1^{-/}$, Masp $2^{-/-}$, or $\mathrm{C}^{-/-}$mice at $37^{\circ} \mathrm{C}$ for 1 hour. Heat-inactivated WT serum was used as a negative control. Cells were washed with PBS, fixed (but not permeabilized), and subjected to immunofluorescence for CL-11 and C3d as described earlier. Alternatively, RTECs cultured from Colec11 $1^{-/}$kidneys were incubated with rCL-11, washed, and then incubated with serum from Colec11 $11^{-/-}$mice, washed again, and then stained for C3d.

Image analysis of CL-11 and C3d. Deposited CL-11 and C3d on cultured RTECs was visualized using an Olympus BX-51 microscope and quantified using ImageJ software (NIH). From each coverslip of stained cultured cells, 7-10 individual fields were photographed. The percentage of positive staining in each image was calculated automatically once the threshold adjustments had been determined to remove background signal.

Confocal microscopy. Confocal scanning electron microscopy was performed using a Nikon Eclipse Ti-E inverted microscope to detect
GATGT-3'; Gapdh: forward, 5'-ACCACAGTCCATGCCATCAC-3' and reverse, 5'-TCCACCACCCTGTTGCTGTA-3'.

Flow cytometry. For detection of endogenous CL-11, single-cell suspensions of human proximal tubular epithelial cells (hPTECs) (a gift of L.C. Racusen, Department of Pathology, Johns Hopkins University School of Medicine, Baltimore, Maryland, USA) were fixed and permeabilized with Cytofix/Cytoperm (BD Biosciences). CL-11 staining was performed by preincubation of cells with mouse anti-CD16-CD32 mAb to block Fc receptors (clone 2.4G2; BD) and then incubated with polyclonal goat anti-human CL-11 Ab (1:100), which cross-reacts with mouse CL-11, followed by donkey anti-goat Alexa Fluor $568 \mathrm{Ab}$. For detection of exogenous CL-11 binding to the cells, single-cell suspensions of hPTECs were incubated with different doses of rCL-11 at $37^{\circ} \mathrm{C}$ for 1 hour, and the cells were then fixed with $4 \%$ paraformaldehyde (but not permeabilized) and subjected to staining for CL-11. Stained cells were analyzed by flow cytometry using a FACSCalibur flow cytometer (BD Biosciences) and FlowJo software.

Statistics. Data are shown as the arithmetic mean \pm SEM or as the readout for individual mice. An unpaired, 2-tailed Student's $t$ test was used for comparisons between 2 groups. A $P$ value of less than 0.05 was considered significant. One- or two-way ANOVA was used for comparisons between three or more groups. All analyses were performed using GraphPad Prism, version 5 (GraphPad Software).

Study approval. The studies performed in animals were approved by the Animal Welfare and Ethical Review Body (AWERB) of King's College London and were in accordance with the United Kingdom Animals (Scientific Procedures) Act of 1986.

\section{Author contributions}

CAF and DT designed and conducted in vivo and in vitro experiments, performed data analysis, and helped write the manuscript. KL contributed to the experimental design and performed in vitro experiments on human tubular cells. WW performed the confocal microscopy. QP cultured cells and performed in vitro 
experiments. WS provided critical review of the manuscript and expertise on the lectin complement pathway. SHS and WZ conceived the study, designed experiments, cosupervised the study, and wrote the manuscript.

\section{Acknowledgments}

This work was supported by Medical Research Council grants MR/J006742/1 (to S. Sacks and W. Zhou) and MR/L020254/1 and G1001141 (to W. Zhou and S. Sacks) and by the National Institute for Health Research (NIHR) Biomedical Research Centre at Guy's and St Thomas' NHS Foundation Trust and King's
College London. D. Tran holds a Complement UK PhD Award sponsored by Alexion Pharmaceuticals Inc. through an educational grant. We thank T. Feizi and S. Hansen for useful discussions and Alma Ayiz for help with statistical analyses. The views expressed are those of the author(s) and not necessarily those of the NHS, the NIHR, or the UK Department of Health.

Address for correspondence: Steven Sacks, MRC Centre for Transplantation, King's College London, 5th Floor Tower Wing, Guy's Hospital, Great Maze Pond, London SE1 9RT, United Kingdom. Phone: 44.0.20.7188.5669; E-mail: steven.sacks@kcl.ac.uk.
1. Pasparakis M. Regulation of tissue homeo-

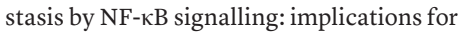
inflammatory diseases. Nat Rev Immunol. 2009;9(11):778-788

2. Holtzman MJ, Byers DE, Alexander-Brett J, Wang X. The role of airway epithelial cells and innate immune cells in chronic respiratory disease. Nat Rev Immunol. 2014;14(10):686-698.

3. Hansen S, et al. Collectin 11 (CL-11, CL-K1) is a MASP-1/3-associated plasma collectin with microbial-binding activity. J Immunol. 2010;185(10):6096-6104.

4. Motomura W, et al. Immunolocalization of a novel collectin CL-K1 in murine tissues. J Histochem Cytochem. 2008;56(3):243-252.

5. Selman L, Hansen S. Structure and function of collectin liver 1 (CL-L1) and collectin 11 (CL-11, CL-K1). Immunobiology. 2012;217(9):851-863.

6 . Thiel S. Complement activating soluble pattern recognition molecules with collagen-like regions, mannan-binding lectin, ficolins and associated proteins. Mol Immunol. 2007;44(16):3875-3888.

7. Waters P, Vaid M, Kishore U, Madan T. Lung surfactant proteins $\mathrm{A}$ and $\mathrm{D}$ as pattern recognition proteins. Adv Exp Med Biol. 2009;653:74-97.

8. Ma YJ, Skjoedt MO, Garred P. Collectin-11/MASP complex formation triggers activation of the lectin complement pathway - the fifth lectin pathway initiation complex. J Innate Immun. 2013;5(3):242-250.

9. van de Wetering JK, van Golde LM, Batenburg JJ. Collectins: players of the innate immune system. Eur J Biochem. 2004;271(7):1229-1249.

10. Holmskov U, Thiel S, Jensenius JC. Collections and ficolins: humoral lectins of the innate immune defense. Annu Rev Immunol. 2003;21:547-578.

11. Keshi $\mathrm{H}$, et al. Identification and characterization of a novel human collectin CL-K1. Microbiol Immunol. 2006;50(12):1001-1013.

12. Selman L, et al. An enzyme-linked immunosorbent assay (ELISA) for quantification of human collectin 11 (CL-11, CL-K1). J Immunol Methods. 2012;375(1-2):182-188.

13. Takahashi K, et al. Elevated plasma CL-K1 level is associated with a risk of developing disseminated intravascular coagulation (DIC). J Thromb Thrombolysis. 2014;38(3):331-338.

14. Yoshizaki T, et al. Comparison of human blood concentrations of collectin kidney 1 and mannan-binding lectin. J Biochem. 2012;151(1):57-64.

15. Maynard Y, Baenziger JU. Characterization of a mannose and $\mathrm{N}$-acetylglucosamine-specific lectin present in rat hepatocytes. J Biol Chem . 1982;257(7):3788-3794.

16. Rooryck C, et al. Mutations in lectin complement pathway genes COLEC11 and MASP1 cause 3MC syndrome. Nat Genet. 2011;43(3):197-203.

17. Peng Q, et al. C3a and C5a promote renal ischemia-reperfusion injury. J Am Soc Nephrol. 2012;23(9):1474-1485.

18. Farrar CA, Zhou W, Lin T, Sacks SH. Local extravascular pool of $\mathrm{C} 3$ is a determinant of postischemic acute renal failure. FASEB J. 2006;20(2):217-226.

19. Patel H, Smith RA, Sacks SH, Zhou W. Therapeutic strategy with a membrane-localizing complement regulator to increase the number of usable donor organs after prolonged cold storage. J Am Soc Nephrol. 2006;17(4):1102-1111.

20. Zhou W, et al. Predominant role for C5b-9 in renal ischemia/reperfusion injury. J Clin Invest. 2000;105(10):1363-1371.

21. Lin F, Moran A, Igarashi P. Intrarenal cells, not bone marrow-derived cells, are the major source for regeneration in postischemic kidney. J Clin Invest. 2005;115(7):1756-1764.

22. Racusen LC, et al. Cell lines with extended in vitro growth potential from human renal proximal tubule: characterization, response to inducers, and comparison with established cell lines. J Lab Clin Med.1997;129(3):318-329.

23. Becker DJ, Lowe JB. Fucose: biosynthesis and biological function in mammals. Glycobiology. 2003;13(7):41R-53R.

24. Biancone L, et al. Alternative pathway activation of complement by cultured human proximal tubular epithelial cells. Kidney Int. 1994;45(2):451-460.

25. David S, Biancone L, Caserta C, Bussolati B, Cambi V, Camussi G. Alternative pathway complement activation induces proinflammatory activity in human proximal tubular epithelial cells. Nephrol Dial Transplant. 1997;12(1):51-56.

26. Schwaeble WJ, et al. Targeting of mannan-binding lectin-associated serine protease- 2 confers protection from myocardial and gastrointestinal ischemia/reperfusion injury. Proc Natl Acad Sci U S A. 2011;108(18):7523-7528.

27. Asgari E, et al. Mannan-binding lectin-associated serine protease 2 is critical for the development of renal ischemia reperfusion injury and mediates tissue injury in the absence of complement $\mathrm{C} 4$. FASEB J. 2014;28(9):3996-4003.

28. Bonventre JV, Yang L. Cellular pathophysiology of ischemic acute kidney injury. J Clin Invest.
2011;121(11):4210-4221.

29. Thurman JM, Ljubanovic D, Edelstein CL, Gilkeson GS, Holers VM. Lack of a functional alternative complement pathway ameliorates ischemic acute renal failure in mice. J Immunol. 2003;170(3):1517-1523.

30. Thurman JM, et al. Treatment with an inhibitory monoclonal antibody to mouse factor B protects mice from induction of apoptosis and renal ischemia/reperfusion injury. J Am Soc Nephrol. 2006;17(3):707-715.

31. Lachmann PJ. The amplification loop of the complement pathways. Adv Immunol. 2009;104:115-149.

32. Takahashi M, et al. Essential role of mannose-binding lectin-associated serine protease-1 in activation of the complement factor $D$. JExp Med. 2010;207(1):29-37.

33. Arend WP, et al. Roles of adipocytes and fibroblasts in activation of the alternative pathway of complement in inflammatory arthritis in mice. J Immunol. 2013;190(12):6423-6433.

34. Henriksen ML, et al. Heteromeric complexes of native collectin kidney 1 and collectin liver 1 are found in the circulation with MASPs and activate the complement system. J Immunol. 2013;191(12):6117-6127.

35. Pratt JR, et al. Nontransgenic hyperexpression of a complement regulator in donor kidney modulates transplant ischemia/reperfusion damage, acute rejection, and chronic nephropathy. Am J Pathol. 2003;163(4):1457-1465.

36. Pratt JR, Basheer SA, Sacks SH. Local synthesis of complement component $\mathrm{C} 3$ regulates acute renal transplant rejection. Nat Med. 2002;8(6):582-587.

37. Laudisi F, et al. Cutting edge: the NLRP3 inflammasome links complement-mediated inflammation and IL-1 $\beta$ release. J Immunol. 2013;191(3):1006-1010.

38. Triantafilou K, Hughes TR, Triantafilou M, Morgan BP. The complement membrane attack complex triggers intracellular $\mathrm{Ca}^{2+}$ fluxes leading to NLRP3 inflammasome activation. J Cell Sci. 2013;126(pt 13):2903-2913.

39. Thurman JM, et al. Altered renal tubular expression of the complement inhibitor Crry permits complement activation after ischemia/reperfusion. J Clin Invest. 2006;116(2):357-368.

40. Tang T, et al. A mouse knockout library for secreted and transmembrane proteins. Nat Biotechnol. 2010;28(7):749-755.

41. Wessels MR, Butko P, Ma M, Warren HB, Lage 
AL, Carroll MC. Studies of group B streptococcal infection in mice deficient in complement component $\mathrm{C} 3$ or $\mathrm{C} 4$ demonstrate an essential role for complement in both innate and acquired immunity. Proc Natl Acad Sci US A.
1995;92(25):11490-11494.

42. Li K, Patel H, Farrar CA, Hargreaves RE, Sacks $\mathrm{SH}$, Zhou W. Complement activation regulates the capacity of proximal tubular epithelial cell to stimulate alloreactive $\mathrm{T}$ cell response. J Am Soc
Nephrol. 2004;15(9):2414-2422.

43. Livak KJ, Schmittgen TD. Analysis of relative gene expression data using real-time quantitative PCR and the 2(-Delta Delta C(T)) Method. Methods. 2001;25(4):402-408. 